\title{
NARROW ORTHOGONALLY ADDITIVE OPERATORS
}

\author{
MARAT PLIEV AND MIKHAIL POPOV
}

\begin{abstract}
We extend the notion of narrow operators to nonlinear maps on vector lattices. The main objects are orthogonally additive operators and, in particular, abstract Uryson operators. Most of the results extend known theorems obtained by O. Maslyuchenko, V. Mykhaylyuk and the second named author published in Positivity 13 (2009), pp. 459-495, for linear operators. For instance, we prove that every orthogonally additive laterally-to-norm continuous C-compact operator from an atomless Dedekind complete vector lattice to a Banach space is narrow. Another result asserts that the set $\mathcal{U}_{o n}^{l c}(E, F)$ of all order narrow laterally continuous abstract Uryson operators is a band in the vector lattice of all laterally continuous abstract Uryson operators from an atomless vector lattice $E$ with the principal projection property to a Dedekind complete vector lattice $F$. The band generated by the disjointness preserving laterally continuous abstract Uryson operators is the orthogonal complement to $\mathcal{U}_{n}^{l c}(E, F)$.
\end{abstract}

\section{INTRODUCTION}

1.1. About the paper. Narrow operators were introduced and studied in 1990 by Plichko and the second named author [14 as a generalization of compact operators defined on symmetric function spaces. Since that, narrow operators were defined on much more general domain spaces generalizing the previous cases, like Köthe function spaces [3], vector lattices [10] and lattice normed spaces [16]. Now it is a subject of an intensive study (see recent monograph [18]).

Some properties of AM-compact operators are generalized to narrow operators, but not all of them. For example, a sum of two narrow operators on $L_{1}$ is narrow, but on an r.i. space $E$ on $[0,1]$ with an unconditional basis every operator is a sum of two narrow operators. Similar questions are very interesting and some of them are involved, see problems [18, Chapter 12] and recent papers [11], [17]. In 2009 O. Maslyuchenko, Mykhaylyuk and the second named author discovered that the sum phenomenon for narrow operators has a pure lattice nature, extending the notion to vector lattices [10]. Namely, a sum of two regular narrow operators is always narrow in a quite general case. Since all operators on $L_{1}$ are regular, the sum of any two narrow operators on $L_{1}$ is narrow. But if $E$ is an r.i. space on $[0,1]$ with

2010 Mathematics Subject Classification. Primary 47H30; Secondary 47H99.

Key words and phrases. Narrow operators, C-compact operators, orthogonally additive operators; abstract Uryson operators, Banach lattices. 
an unconditional basis then all examples of pairs of narrow operators with nonnarrow sum involve non-regular operators.

In the present paper we generalize the main results of [10] on narrow operators to a wide class including non-linear maps called orthogonally additive operators. Our main observation here is that, in the most contexts of narrow operators we actually use their linearity only on orthogonal elements. On the other hand, all necessary background for such operators already has been built [12, [13].

Formally, the definitions of narrow and order narrow operators on vector lattices given in [10] can be also applied to nonlinear maps. However, some natural "small" nonlinear maps which have to be narrow by the idea of narrowness, are nonnarrow with respect to these definitions. In this paper, we reformulate the definitions of narrow and order narrow operators in such a way that the new definitions are equivalent to the known ones for linear maps, and are substantial for nonlinear maps.

Using these ideas we prove some new results for orthogonally additive operators and abstract Uryson operators, an important subclass of orthogonally additive operators. Theorem 3.2 asserts that every orthogonally additive laterally-to-norm continuous $C$-compact operator from an atomless Dedekind complete vector lattice to a Banach space is narrow. In Theorem 4.1 we solve a dominated problem for order narrow abstract Uryson operators: if $E, F$ are vector lattices with $E$ atomless and $F$ an ideal of some order continuous Banach lattice then every abstract Uryson operator $T: E \rightarrow F$ is order narrow if and only if $|T|$ is. Then we prove a number of statements which concern auxiliary notions of $\lambda$-narrow and pseudonarrow operators, the main of which theorems 9.1 and 9.2 establish that all the notions of narrow operators coincide, under some not so restrictive assumptions. Moreover, Theorem 9.2 asserts that the set $\mathcal{U}_{\text {onlc }}(E, F)$ of all order narrow laterally continuous abstract Uryson operators is a band in the Dedekind complete vector lattice of all laterally continuous abstract Uryson operator from $E$ to $F$. Moreover, the orthogonal complement to $\mathcal{U}_{\text {onlc }}(E, F)$ equals the band generated by all disjointness preserving laterally continuous abstract Uryson operators from $E$ to $F$.

Ideologically, all the proofs from [10] remain almost the same for the corresponding results in the present paper. However, pretty much all of them needed for reconstruction, and several (especially Lemma 6.3) needed for a new approach.

1.2. Terminology and notation. General information on vector lattices and Banach spaces the reader can find in the books [1, 5, 8, 9].

Let $E$ be a vector lattice. An element $u>0$ of $E$ is an atom provided the conditions $0 \leq x \leq u, 0 \leq y \leq u$ and $x \wedge y=0$ imply that either $x=0$ or $y=0$. A vector lattice is said to be atomless provided it has no atom. An element $y$ of a vector lattice $E$ is called a fragment (in another terminology, a component) of an element $x \in E$, provided $y \perp(x-y)$. The notation $y \sqsubseteq x$ 
means that $y$ is a fragment of $x$. Evidently, a non-zero element $x \in E$ is an atom if and only if the only fragments of $x$ are 0 and $x$ itself. Hence, a vector lattice $E$ is atomless if each non-zero element $x \in E$ has a proper fragment $y \sqsubseteq x$, that is, $0 \neq y \neq x$. A net $\left(x_{\alpha}\right)_{\alpha \in \Lambda}$ in $E$ order converges to an element $x \in E$ (notation $x_{\alpha} \stackrel{\mathrm{o}}{\longrightarrow} x$ ) if there exists a net $\left(u_{\alpha}\right)_{\alpha \in \Lambda}$ in $E$ such that $u_{\alpha} \downarrow 0$ and $\left|x_{\beta}-x\right| \leq u_{\beta}$ for all $\beta \in \Lambda$. The equality $x=\bigsqcup_{i=1}^{n} x_{i}$ means that $x=\sum_{i=1}^{n} x_{i}$ and $x_{i} \perp x_{j}$ if $i \neq j$. Two fragments $x_{1}, x_{2}$ of $x$ are called mutually complemented or $M C$, in short, if $x=x_{1} \sqcup x_{2}$. A sequence $\left(e_{n}\right)_{n=1}^{\infty}$ is called a disjoint tree on $e \in E$ if $e_{1}=e$ and $e_{n}=e_{2 n} \sqcup e_{2 n+1}$ for each $n \in \mathbb{N}$. It is clear that all $e_{n}$ are fragments of $e$.

Fix a vector lattice $E$ and $e \in E$. The set of the all fragments of $e$ we denote by $\mathfrak{F}_{e}$. Denote by $\Pi_{e}$ the collection of all finite subsets $\pi \subset \mathfrak{F}_{e}$ such that $e=\bigsqcup_{x \in \pi} x$. For $\pi_{1}, \pi_{2} \in \Pi_{e}$ we write $\pi_{1} \leq \pi_{2}$ provided for every $y \in \pi_{2}$ there exists $x \in \pi_{1}$ such that $y \sqsubseteq x$. Observe that $\pi_{1} \leq \pi_{2}$ if and only if for every $x \in \pi_{1}$ there exists a subset $\pi \subseteq \pi_{2}$ such that $x=\bigsqcup_{y \in \pi} y$. Note that $\Pi_{e}$ is a directed set: given any $\pi_{1}, \pi_{2} \in \Pi_{e}$, one has that $\pi_{1} \leq \pi_{3}$ and $\pi_{2} \leq \pi_{3}$, where $\pi_{3}=\left\{x \wedge y: x \in \pi_{1} \& y \in \pi_{2}\right\}$.

1.3. Narrow operators. The following two definitions of a narrow operator were given in [10], depending on whether the range space is a Banach space or a vector lattice.

Definition 1.1. Let $E$ be an atomless vector lattice and $X$ a Banach space. A map $f: E \rightarrow X$ is called

- narrow, if for every $x \in E^{+}$and every $\varepsilon>0$ there exists $y \in E$ such that $|y|=x$ and $\|f(y)\|<\varepsilon$;

- strictly narrow, if for every $x \in E^{+}$there exists $y \in E$ such that $|y|=x$ and $f(y)=0$.

Definition 1.2. Let $E, F$ be vector lattices with $E$ atomless. A linear operator $T: E \rightarrow F$ is called order narrow if for every $x \in E^{+}$there exists a net $\left(x_{\alpha}\right)$ in $E$ such that $\left|x_{\alpha}\right|=x$ for each $\alpha$, and $T x_{\alpha} \stackrel{\circ}{\rightarrow} 0$.

The atomless condition on $E$ is not essential in the above two definitions, however a narrow map must send atoms to zero, so the condition serves to avoid triviality.

1.4. Orthogonally additive operators. This class of operators acting between vector lattices was introduced and studied in 1990 by Mazón and Segura de León [12, 13], and then considered to be defined on lattice-normed spaces by Kusraev and Pliev [6, 7, 15]. Let $E$ be a vector lattice and $F$ a real linear space. An operator $T: E \rightarrow F$ is called orthogonally additive if $T(x+y)=T(x)+T(y)$ whenever $x, y \in E$ are disjoint. It follows from the definition that $T(0)=0$. It is immediate that the set of all orthogonally additive operators is a real vector space with respect to the natural linear operations. 
Let $E$ and $F$ be vector lattices. An orthogonally additive operator $T$ : $E \rightarrow F$ is called:

- positive if $T x \geq 0$ holds in $F$ for all $x \in E$;

- order bounded it $T$ maps order bounded sets in $E$ to order bounded sets in $F$.

Observe that if $T: E \rightarrow F$ is a positive orthogonally additive operator and $x \in E$ is such that $T(x) \neq 0$ then $T(-x) \neq-T(x)$, because otherwise both $T(x) \geq 0$ and $T(-x) \geq 0$ imply $T(x)=0$. So, the above notion of positivity is far from the usual positivity of a linear operator: the only linear operator which is positive in the above sense is zero.

A positive orthogonally additive operator need not be order bounded. Indeed, every function $T: \mathbb{R} \rightarrow \mathbb{R}$ with $T(0)=0$ is an orthogonally additive operator, and obviously, not each of them is order bounded.

Another useful observation is that, if $T: E \rightarrow F$ is a positive orthogonally additive operator and $x \sqsubseteq y \in E$ then $T(x) \leq T(y)$, no matter whether $y$ is positive or not.

1.5. Abstract Uryson operators. The classical integral abstract Uryson operator is presented in the following example.

Example 1. Let $(A, \Sigma, \mu)$ and $(B, \Xi, \nu)$ be $\sigma$-finite complete measure spaces, and let $(A \times B, \mu \times \nu)$ denote the completion of their product measure space. Let $K: A \times B \times \mathbb{R} \rightarrow \mathbb{R}$ be a function satisfying the following conditions:

$\left(C_{0}\right) K(s, t, 0)=0$ for $\mu \times \nu$-almost all $(s, t) \in A \times B$;

$\left(C_{1}\right) K(\cdot, \cdot, r)$ is $\mu \times \nu$-measurable for all $r \in \mathbb{R}$;

$\left(C_{2}\right) K(s, t, \cdot)$ is continuous on $\mathbb{R}$ for $\mu \times \nu$-almost all $(s, t) \in A \times B$.

Given $f \in L_{0}(A, \Sigma, \mu)$, the function $|K(s, \cdot, f(\cdot))|$ is $\mu$-measurable for $\nu$ almost all $s \in B$ and $h_{f}(s):=\int_{A}|K(s, t, f(t))| d \mu(t)$ is a well defined and $\nu$-measurable function. Since the function $h_{f}$ can be infinite on a set of positive measure, we define

$$
\operatorname{Dom}_{A}(K):=\left\{f \in L_{0}(\mu): h_{f} \in L_{0}(\nu)\right\} .
$$

Then we define an operator $T: \operatorname{Dom}_{A}(K) \rightarrow L_{0}(\nu)$ by setting

$$
(T f)(s):=\int_{A} K(s, t, f(t)) d \mu(t) \nu \text { - a.e. }(\star)
$$

Let $E$ and $F$ be order ideals in $L_{0}(\mu)$ and $L_{0}(\nu)$ respectively, $K$ a function satisfying $\left(C_{0}\right)-\left(C_{2}\right)$. Then $(\star)$ defines an orthogonally additive order bounded integral operator acting from $E$ to $F$ if $E \subseteq \operatorname{Dom}_{A}(K)$ and $T(E) \subseteq F$.

In [12] Mazón and Segura de León introduced and studied abstract Uryson operators, that possess the main properties of the integral Uryson operators. More precisely, an orthogonally additive order bounded operator $T: E \rightarrow F$

\footnotetext{
${ }^{1}\left(C_{1}\right)$ and $\left(C_{2}\right)$ are called the Carathéodory conditions
} 
between vector lattices $E, F$ is called an abstract Uryson operator. For example, any linear operator $T \in L_{+}(E, F)$ defines a positive abstract Uryson operator by $G(f)=T|f|$ for each $f \in E$. The set of all abstract Uryson operators from $E$ to $F$ we denote by $\mathcal{U}(E, F)$. Consider some more examples of abstract Uryson operators.

Example 2. We consider the vector space $\mathbb{R}^{m}, m \in \mathbb{N}$ as a vector lattice with the coordinate-wise order: for any $x, y \in \mathbb{R}^{m}$ we set $x \leq y$ provided $e_{i}^{*}(x) \leq e_{i}^{*}(y)$ for all $i=1, \ldots, m$, where $\left(e_{i}^{*}\right)_{i=1}^{m}$ is the coordinate functionals on $\mathbb{R}^{m}$. Let $T: \mathbb{R}^{n} \rightarrow \mathbb{R}^{m}$. Then $T \in \mathcal{U}\left(\mathbb{R}^{n}, \mathbb{R}^{m}\right)$ if and only if there are real functions $T_{i, j}: \mathbb{R} \rightarrow \mathbb{R}, 1 \leq i \leq m, 1 \leq j \leq n$ satisfying $T_{i, j}(0)=0$ such that

$$
e_{i}^{*}\left(T\left(x_{1}, \ldots, x_{n}\right)\right)=\sum_{j=1}^{n} T_{i, j}\left(x_{j}\right),
$$

In this case we write $T=\left(T_{i, j}\right)$.

Example 3. Let $T: l^{2} \rightarrow \mathbb{R}$ be the operator defined by

$$
T\left(x_{1}, \ldots, x_{n}, \ldots\right)=\sum_{n \in I_{x}} n\left(\left|x_{n}\right|-1\right)
$$

where $I_{x}:=\left\{n \in \mathbb{N}:\left|x_{n}\right| \geq 1\right\}$. It is not difficult to check that $T$ is a positive abstract Uryson operator.

Example 4. Let $(\Omega, \Sigma, \mu)$ be a measure space, $E$ a sublattice of the vector lattice $L_{0}(\mu)$ of all equivalence classes of $\Sigma$-measurable functions $x: \Omega \rightarrow \mathbb{R}$, $F$ a vector lattice and $\nu: \Sigma \rightarrow F$ a finitely additive measure. Then the map $T: E \rightarrow F$ given by $T(x)=\nu(\operatorname{supp} x)$ for any $x \in E$, is an abstract Uryson operator which is positive if and only if $\nu$ is positive.

The set of all abstract Uryson operators from $E$ to $F$ we denote by $\mathcal{U}(E, F)$. Consider the following order on $\mathcal{U}(E, F): S \leq T$ whenever $T-S$ is a positive operator. Then $\mathcal{U}(E, F)$ becomes an ordered vector space. Actually, one can prove more.

Theorem 1.3. ([12, Theorem 3.2.). Let $E$ and $F$ be vector lattices, $F$ Dedekind complete. Then $\mathcal{U}(E, F)$ is a Dedekind complete vector lattice. Moreover, for each $S, T \in \mathcal{U}(E, F)$ and $x \in E$ the following conditions hold

(1) $(T \vee S)(x)=\sup \{T(y)+S(z): x=y \sqcup z\}$.

(2) $(T \wedge S)(x)=\inf \{T(y)+S(z): x=y \sqcup z\}$.

(3) $(T)^{+}(x)=\sup \{T y: y \sqsubseteq x\}$.

(4) $(T)^{-}(x)=-\inf \{T y: y \sqsubseteq x\}$.

(5) $|T(x)| \leq|T|(x)$.

We also need the following result which represents the lattice operations of $\mathcal{U}(E, F)$ in terms of directed systems.

Theorem 1.4 ([13, Lemma 3.2). Let $E$ and $F$ be vector lattices, $F$ Dedekind complete. Then for all $T, S \in \mathcal{U}(E, F)$ and $x \in E$ we have that 
(1) $\left\{\sum_{i=1}^{n} T\left(y_{i}\right) \wedge S\left(y_{i}\right): x=\bigsqcup_{i=1}^{n} y_{i} ; n \in \mathbb{N}\right\} \downarrow(S \wedge T)(x)$.

(2) $\left\{\sum_{i=1}^{n} T\left(y_{i}\right) \vee S\left(y_{i}\right): x=\bigsqcup_{i=1}^{n} y_{i} ; n \in \mathbb{N}\right\} \uparrow(S \vee T)(x)$.

(3) $\left\{\sum_{i=1}^{n}\left|T\left(y_{i}\right)\right|: x=\bigsqcup_{i=1}^{n} y_{i} ; n \in \mathbb{N}\right\} \uparrow|T|(x)$.

\section{Definitions of NARROW AND ORDER NARROW OPERATORS FOR ORTHOGONALLY ADDITIVE MAPS}

Let $(\Omega, \Sigma, \mu)$ be a finite atomless measure space, $1 \leq p<\infty$ and let $T: L_{p}(\mu) \rightarrow \mathbb{R}$ be the map defined by

$$
T x=\|x\|^{p} \text { for all } x \in L_{p}(\mu) .
$$

Observe that $T$ is a nonnarrow abstract Uryson operator with respect to Definition 1.1. On the other hand, by the initial idea, narrow operator must contain "small" operators like the above, which is a rank one operator. The same properties possesses the operator from Example 4. The first example of a nonnarrow continuous linear functional was provided in [10. However, this functional is not order-to-norm continuous and defined on $L_{\infty}$, the norm of which is not absolutely continuous (order continuous). To the contrast, the operator $T$ defined by (2.1) is order-to-norm continuous. The following definition, being equivalent to Definition 1.1 for linear maps, makes this operator to be narrow.

Definition 2.1. Let $E$ be an atomless vector lattice and $X$ a Banach space. A map $T: E \rightarrow X$ is called:

- narrow at a point $e \in E$ if for every $\varepsilon>0$ there exist $M C$ fragments $e_{1}, e_{2}$ of $e$ such that $\left\|T\left(e_{1}\right)-T\left(e_{2}\right)\right\|<\varepsilon$;

- narrow if it is narrow at every $e \in E$;

- locally narrow at $e_{0}$ if $T$ is narrow at every $e \in \mathcal{F}_{e_{0}}$;

- strictly narrow at $e \in E$ if there exist $M C$ fragments $e_{1}, e_{2}$ of $e$ such that $T\left(e_{1}\right)=T\left(e_{2}\right)$;

- strictly narrow if it is strictly narrow at every $e \in E$.

Next is the corresponding new definition of an order narrow operator.

Definition 2.2. Let $E, F$ be vector lattices with $E$ atomless. A map $T$ : $E \rightarrow F$ is called

- order narrow at a point $e \in E$ if there exists a net of decompositions $e=f_{\alpha} \sqcup g_{\alpha}$ such that $\left(T\left(f_{\alpha}\right)-T\left(g_{\alpha}\right)\right) \stackrel{\mathrm{o}}{\longrightarrow} 0$;

- order narrow if it is order narrow at every $e \in E$;

- locally order narrow at $e_{0}$ if $T$ is order narrow at every $e \in \mathcal{F}_{e_{0}}$.

We will use the following simple observation.

Proposition 2.3. Let $E$ be an atomless vector lattice and $X$ a Banach space (resp., vector space or vector lattice). If an orthogonally additive operator 
$T: E \rightarrow X$ is narrow (resp., strictly narrow or order narrow) at every $e \in E^{+} \cup E^{-}$then $T$ is narrow (resp., strictly narrow or order narrow).

Proof. We prove for the case of a narrow operator; proofs for the rest of cases are similar. Fix any $e \in E$ and $\varepsilon>0$. Choose decompositions $e^{+}=e_{1} \sqcup e_{2}$ $-e^{-}=e_{1}^{\prime} \sqcup e_{2}^{\prime}$ so that $\left\|T\left(e_{1}\right)-T\left(e_{2}\right)\right\|<\frac{\varepsilon}{2}$ and $\left\|T\left(e_{1}^{\prime}\right)-T\left(e_{2}^{\prime}\right)\right\|<\frac{\varepsilon}{2}$. Then $e=\left(e_{1}+e_{1}^{\prime}\right) \sqcup\left(e_{2}+e_{2}^{\prime}\right)$ and

$$
\begin{aligned}
\left\|T\left(e_{1}+e_{1}^{\prime}\right)-T\left(e_{2}+e_{2}^{\prime}\right)\right\| & =\left\|T\left(e_{1}\right)+T\left(e_{1}^{\prime}\right)-T\left(e_{2}\right)-T\left(e_{2}^{\prime}\right)\right\| \\
& \leq\left\|T\left(e_{1}\right)-T\left(e_{2}\right)\right\|+\left\|T\left(e_{1}^{\prime}\right)-T\left(e_{2}^{\prime}\right)\right\|<\varepsilon .
\end{aligned}
$$

Remark that narrowness of an orthogonally additive operator $T$ at elements $e \in E^{+}$does not imply narrowness $T$ at elements $e \in E^{-}$. Indeed, given any orthogonally additive operators $T_{1}, T_{2}: E \rightarrow X$, the operator $T: E \rightarrow X$ defined by $T(x)=T_{1}\left(x^{+}\right)+T_{2}\left(-x^{-}\right)$for all $x \in E$ is orthogonally additive. So, for $T_{1}=0$ and $T_{2}=-I d$, where $I d$ is the identity operator on $E$, the orthogonally additive operator given by $T(x)=x^{-}$for all $x \in E$ is narrow at all positive elements and is not narrow at all strictly negative elements.

Proofs of the following two lemmas are simple exercises.

Lemma 2.4. Let $E, F$ be vector lattices with $E$ atomless. A linear operator $T: E \rightarrow F$ is narrow (strictly narrow) in the sense of Definition 1.1 if and only if $T$ is narrow (strictly narrow) in the sense of Definition [2.1.

Lemma 2.5. Let $E, F$ be vector lattices with $E$ atomless. A linear operator $T: E \rightarrow F$ is order narrow in the sense of Definition 1.2 if and only if $T$ is order narrow in the sense of Definition 2.2.

The proof of the following lemma is almost the same as the proof of the corresponding statement for linear maps (see [16, Lemma 3.2] and [18, Proposition 10.7]).

Lemma 2.6. Let $E$ be an atomless vector lattice and $F$ a Banach lattice. Then every narrow abstract Uryson operator $T: V \rightarrow W$ is order narrow.

The sets of narrow and order narrow abstract Uryson operators coincide if the range vector lattice is good enough.

Lemma 2.7. Let $E$ be an atomless vector lattice and $F$ a Banach lattice with an order continuous norm. Then an abstract Uryson operator $T: E \rightarrow F$ is order narrow if and only if $T$ is narrow.

Proof. Let $T$ be order narrow. Then for every $e \in E$ there exists a net of decompositions $e=f_{\alpha} \sqcup g_{\alpha}, \alpha \in \Lambda$ such that $\left(T\left(f_{\alpha}\right)-T\left(g_{\alpha}\right)\right) \stackrel{\mathrm{o}}{\longrightarrow} 0$. Fix any $\varepsilon>0$. By the order continuity of the norm of $F$, we can find $\alpha_{0} \in \Lambda$ such that $\left\|T\left(f_{\alpha}\right)-T\left(g_{\alpha}\right)\right\|<\varepsilon$ for every $\alpha \geq \alpha_{0}$. So, $T$ is narrow. In view of Lemma 2.6, the converse is true. 
Lemma 2.8. Let $E, F$ and $G$ be vector lattices with $E$ atomless and $F$ an order ideal of $G$. If an abstract Uryson operator $T: E \rightarrow F$ is order narrow (resp., locally order narrow) at a point $x \in E$ then $T: E \rightarrow G$ is order narrow (resp., locally order narrow) at $x$. Conversely, if an abstract Uryson operator $T: E \rightarrow F$ is such that $T: E \rightarrow G$ is order narrow (resp., locally order narrow) at a point $x \in E$ then so is $T: E \rightarrow F$.

Proof. Observe that the statement for locally order narrow operators follows from the same statement for order narrow operators, so we prove it for order narrow operators. The first part is obvious. Let $T: E \rightarrow F$ be an abstract Uryson operator such that $T: E \rightarrow G$ is order narrow at $x_{0} \in E$ and $x \in \mathcal{F}_{x_{0}}$. Choose a net of decompositions $x=y_{\alpha} \sqcup z_{\alpha}, \alpha \in \Lambda$ so that $\left(T\left(y_{\alpha}\right)-T\left(z_{\alpha}\right)\right) \stackrel{\mathrm{o}}{\longrightarrow} 0$ in $G$, that is, $\left|T\left(y_{\alpha}\right)-T\left(z_{\alpha}\right)\right| \leq u_{\alpha} \downarrow 0$ for some net $\left(u_{\alpha}\right)_{\alpha \in \Lambda}$ in $G$. By the order boundedness of $T$, there is $f \in F$ such that $|T y| \leq f$ for every $y \sqsubseteq x$. In particular, $\left|T\left(y_{\alpha}\right)-T\left(z_{\alpha}\right)\right| \leq 2 f$. Hence, $\left|T\left(y_{\alpha}\right)-T\left(z_{\alpha}\right)\right| \leq w_{\alpha} \downarrow 0$ where $w_{\alpha}=(2 f) \wedge u_{\alpha} \in F$. So, we have that $\left(w_{\alpha}\right)_{\alpha \in \Lambda} \subseteq G$. Thus, $\left(T\left(y_{\alpha}\right)-T\left(z_{\alpha}\right)\right)_{\alpha \in \Lambda} \stackrel{\mathrm{o}}{\longrightarrow} 0$ in $F$.

\section{LATERALly-TO-NORM CONTINUOUS C-COMPACT ABSTRACT URYSON OPERATORS ARE NARROW}

In this section we generalize the result of O. Maslyuchenko, Mykhaylyuk and the second named author [10] that every order-to-norm continuous AM-compact (linear) operator is narrow (see also [18, Theorem 10.17]). Firstly, we consider orthogonally additive operators which is more general than linear operators. Secondly, laterally-to-norm continuity is weaker than order-to-norm continuity. Finally, C-compactness is weaker than the AMcompactness.

Recall that a net $\left(x_{\alpha}\right)$ in a vector lattice $E$ laterally converges to $x \in E$ if $x_{\alpha} \sqsubseteq x_{\beta} \sqsubseteq x$ for all indices $\alpha<\beta$ and $x_{\alpha} \stackrel{\mathrm{o}}{\longrightarrow} x$. In this case we write $x_{\alpha} \stackrel{\text { lat }}{\longrightarrow} x$. For positive elements $x_{\alpha}, x$ the condition $x_{\alpha} \stackrel{\text { lat }}{\longrightarrow} x$ means that $x_{\alpha} \sqsubseteq x$ and $x_{\alpha} \uparrow x$.

Definition 3.1. Let $E$ be a vector lattice and $X$ a Banach space. An orthogonally additive operator $T: E \rightarrow X$ is called:

(1) order-to-norm continuous if $T$ sends order convergent nets in $E$ to norm convergent nets in $X$;

(2) laterally-to-norm continuous provided $T$ sends laterally convergent nets in $E$ to norm convergent nets in $X$;

(3) AM-compact if for every order bounded set $M \subset E$ its image $T(M)$ is a relatively compact set in $X$;

(4) $C$-compact if the set $\{T(y): y \sqsubseteq x\}$ is relatively compact in $X$ for every $x \in E$.

Since the order convergence implies the lateral convergence of a net, orderto-norm continuity of a map yields its laterally-to-norm continuity. The 
converse is not true: the abstract Uryson operator $T$ from Example 4 is laterally-to-norm continuous if $\nu$ is a countably additive measure but not order-to-norm continuous if $\nu \neq 0$.

Example 5. Let $\left([0,1], \Sigma_{1}, \mu\right)$ and $\left([0,1], \Sigma_{2}, \nu\right)$ be two measure spaces, $E=C[0,1]$, which is a sublattice of $L_{\infty}(\mu)$, and $F=L_{\infty}(\nu)$. Consider the integral Uryson operator $T: E \rightarrow F$ with the kernel $K(s, t, r)=$ $\mathbf{1}_{[0,1]}(t) \mathbf{1}_{[0,1]}(s)|r|$. Since the interval $[0,1]$ is connected, every numerical continuous function $f:[0,1] \rightarrow \mathbb{R}$ is an atom, that is, $f$ has no nonzero fragment and therefore $\{T(g): g \sqsubseteq f\}$ is a relatively compact set in $F$ for every $f \in E$. Take $u(t)=\mathbf{1}_{[0,1]}(t)$ and consider the order bounded set $D=\{f \in E:|f| \leq u\}$ in $E$. Then we have

$$
T(f)(s)=\int_{0}^{1} \mathbf{1}_{[0,1]}(t) \mathbf{1}_{[0,1]}(s)|f(t)| d \mu(t)=\mathbf{1}_{[0,1]}(s) \int_{0}^{1}|f(t)| d \mu(t) .
$$

Observe that $T(D)$ is not relatively compact in $F$. Therefore $T$ is $C$ compact, but not $A M$-compact.

Remark that a C-compact abstract Uryson operator $T: E \rightarrow F$ between Banach lattices $E, F$ with $F \sigma$-Dedekind complete is AM-compact if, in addition, $T$ is uniformly continuous on order bounded subsets of $E$ [13, Theorem 3.4].

The main result of the section is the following theorem.

Theorem 3.2. Let $E$ be an atomless Dedekind complete vector lattice and $F$ a Banach space. Then every orthogonally additive laterally-to-norm continuous $C$-compact operator $T: E \rightarrow F$ is narrow.

The proof of Theorem 3.2 mainly repeats the proof of [10, Theorem 5.1]. However, now we deal with nonlinear maps, and thus we need to follow it carefully. The following lemma is known as the lemma on rounding off coefficients [4, p. 14].

Lemma 3.3. Let $\left(x_{i}\right)_{i=1}^{n}$ be a finite collection of vectors in a finite dimensional normed space $F$ and let $\left(\lambda_{i}\right)_{i=1}^{n}$ be a collection of reals with $0 \leq \lambda_{i} \leq 1$ for each $i$. Then there exists a collection $\left(\theta_{i}\right)_{i=1}^{n}$ of numbers $\theta_{i} \in\{0,1\}$ such that

$$
\left\|\sum_{i=1}^{n}\left(\lambda_{i}-\theta_{i}\right) x_{i}\right\| \leq \frac{\operatorname{dim} X}{2} \max _{i}\left\|x_{i}\right\| .
$$

Lemma 3.4. Let $E$ be an atomless Dedekind complete vector lattice, $F$ a Banach space, $T: E \rightarrow F$ an orthogonally additive laterally-to-norm continuous operator. If $e \in E,\left|e_{n}\right| \leq|e|$ and $e_{n} \perp e_{m}$ for each integers $n \neq m$ then $\lim _{n \rightarrow \infty}\left\|T\left(e_{n}\right)\right\|=0$.

Proof. Since $E$ is Dedekind complete, the sequence $f_{n}=\sum_{k=1}^{n} e_{k}$ laterally converges to $f=\sum_{k=1}^{\infty} e_{k}$ Then the laterally-to-norm continuity of $T$ implies 
that $T f_{n}$ converges to $T f$ in $\mathrm{F}$. The sequence $\left(T\left(f_{n}\right)\right)_{n=1}^{\infty}$ is fundamental, hence

$$
\left\|T\left(f_{n}\right)-T\left(f_{n-1}\right)\right\|=\left\|T\left(\sum_{k=1}^{n} e_{k}\right)-T\left(\sum_{k=1}^{n-1} e_{k}\right)\right\|=\left\|T\left(e_{n}\right)\right\|
$$

implies $\lim _{n \rightarrow \infty}\left\|T\left(e_{n}\right)\right\|=0$.

Lemma 3.5. Let $E$ be an atomless Dedekind complete vector lattice, $F$ a Banach space, $T: E \rightarrow F$ an orthogonally additive laterally-to-norm continuous operator, $e \in E$. Then there exist $M C$ fragments $e_{1}, e_{2}$ of e such that $\left\|T\left(e_{1}\right)\right\|=\left\|T\left(e_{2}\right)\right\|$.

Proof. Fix any $M C$ fragments $e_{1}, e_{2}$ of $e$. If $\left\|T\left(e_{1}\right)\right\|=\left\|T\left(e_{2}\right)\right\|$ then there is nothing to prove. With no loss of generality we may and do assume that $\left\|T\left(e_{1}\right)\right\|-\left\|T\left(e_{2}\right)\right\|>0$. Consider the partially ordered set

$$
D=\left\{g \sqsubseteq e_{1}:\left\|T\left(e_{1}-g\right)\right\|-\left\|T\left(e_{2}+g\right)\right\| \geq 0\right\}
$$

where $g_{1} \leq g_{2}$ if and only if $g_{1} \sqsubseteq g_{2}$. If $B \subseteq D$ is a chain then $g^{\star}=\vee B \in D$ by the laterally-to-norm continuity of $T$. By the Zorn lemma, there is a maximal element $g_{0} \in D$. Now we show that $\left\|T\left(e_{1}-g_{0}\right)\right\|-\left\|T\left(e_{2}+g_{0}\right)\right\|=0$. Suppose on the contrary that

$$
\alpha=\left\|T\left(e_{1}-g_{0}\right)\right\|-\left\|T\left(e_{2}+g_{0}\right)\right\|>0 .
$$

Since $E$ is atomless, we can choose a fragment $0 \neq f \sqsubseteq\left(e_{1}-g_{0}\right)$ such that $\|T(f)\|<\frac{\alpha}{4}$ and $\|T(-f)\|<\frac{\alpha}{4}$. Since $g_{0} \perp f, g_{0}+f \sqsubseteq e_{1}$ we have

$$
\begin{aligned}
& \left\|T\left(e_{1}-g_{0}-f\right)\right\|-\left\|T\left(e_{2}+g_{0}+f\right)\right\|= \\
& \left\|T\left(e_{1}-g_{0}\right)+T(-f)\right\|-\left\|T\left(e_{2}+g_{0}\right)+T(f)\right\| \geq \\
& \left\|T\left(e_{1}-g_{0}\right)\right\|-\|T(-f)\|+\left\|T\left(e_{2}+g_{0}\right)\right\|-\|T(f)\|>\frac{\alpha}{2}>0,
\end{aligned}
$$

that contradicts the maximality of $g_{0}$.

Lemma 3.6. Let $E$ be an atomless Dedekind complete vector lattice, $F$ a Banach space, $T: E \rightarrow F$ an orthogonally additive laterally-to-norm continuous operator, $e \in E$ and $\left(e_{n}\right)_{n=1}^{\infty}$ be a disjoint tree on e. If $\left\|T\left(e_{2 n}\right)\right\|=$ $\left\|T\left(e_{2 n+1}\right)\right\|$ for every $n \geq 1$ then

$$
\lim _{m \rightarrow \infty} \gamma_{m}=0, \text { where } \gamma_{m}=\max _{2^{m} \leq i<2^{m+1}}\left\|T\left(e_{i}\right)\right\| .
$$

Proof. Set $\varepsilon=\limsup _{m \rightarrow \infty} \gamma_{m}$ and prove that $\varepsilon=0$, which will be enough for the proof. Suppose on the contrary that $\varepsilon>0$. Then for each $n \in \mathbb{N}$ we set

$$
\varepsilon_{n}=\limsup _{m \rightarrow \infty} \max _{2^{m} \leq i<2^{m+1}, e_{i} \sqsubseteq e_{n}}\left\|T\left(e_{i}\right)\right\| .
$$

Hence, for each $m \in \mathbb{N}$ one has

$$
\max _{2^{m} \leq i<2^{m+1}} \varepsilon_{i}=\varepsilon
$$


Now we are going to construct a sequence of mutually disjoint elements $\left(e_{n_{j}}\right)_{j=1}^{\infty}$ such that $\left\|T\left(e_{n_{j}}\right)\right\| \geq \frac{\varepsilon}{2}$, that is impossible by Lemma 3.4. At the first step we choose $m_{1}$ so that $\max _{2^{m_{1}} \leq i<2^{m_{1}+1}}\left\|T\left(e_{i}\right)\right\| \geq \frac{\varepsilon}{2}$. By $(\star)$, we choose $i_{1}, 2^{m_{1}} \leq i_{1}<2^{m_{1}+1}$ so that $\varepsilon_{i_{1}}=\varepsilon$. Using $\left\|T\left(e_{2 n}\right)\right\|=\left\|T\left(e_{2 n+1}\right)\right\|$, we choose $n_{1} \neq i_{1}, 2^{m_{1}} \leq n_{1}<2^{m_{1}+1}$ so that $\left\|T\left(e_{n_{1}}\right)\right\| \geq \frac{\varepsilon}{2}$. At the second step we choose $m_{2}>m_{1}$ so that

$$
\max _{2^{m} \leq i<2^{m+1}, e_{i} \sqsubseteq e_{i_{1}}}\left\|T\left(e_{i}\right)\right\| \geq \frac{\varepsilon}{2} .
$$

By $(\star)$, we choose $i_{2}, 2^{m_{2}} \leq i_{2}<2^{m_{2}+1}$ so that $\varepsilon_{i_{2}}=\varepsilon$. Then we choose $m_{2} \neq i_{2}, 2^{m_{2}} \leq i_{2}<2^{m_{2}+1}$ so that $\left\|T\left(e_{m_{2}}\right)\right\| \geq \frac{\varepsilon}{2}$. Proceeding further, we construct the desired sequence. Indeed, $\left\|T\left(e_{m_{i}}\right)\right\| \geq \frac{\varepsilon}{2}$ by the construction and the mutual disjointness for $e_{m_{l}}, e_{m_{j}}, j \neq l$ is guaranteed by the condition $m_{j} \neq i_{j}$, because the elements $e_{m_{j+l}}$ are fragments of $e_{i_{j}}$ which are disjoint to $e_{m_{j}}$.

Lemma 3.7. Let $E$ be an atomless Dedekind complete vector lattice, $F$ a finite dimensional Banach space. Then every orthogonally additive laterallyto-norm continuous operator $T: E \rightarrow F$ is narrow.

Proof. Fix any $e \in E$ and $\varepsilon>0$. Using Lemma 3.5, we construct a disjoint tree $\left(e_{n}\right)$ on $e$ with $\left\|T\left(e_{2 n}\right)\right\|=\left\|T\left(e_{2 n+1}\right)\right\|$ for all $n \in \mathbb{N}$. By lemma 3.6 we choose $m$ so that $\gamma_{m} \operatorname{dim} F<\varepsilon$. Then using Lemma 3.3, we choose numbers $\lambda_{i} \in\{0,1\}$ for $i=2^{m}, \ldots, 2^{m+1}-1$ so that

$$
\left\|2 \sum_{i=2^{m}}^{2^{m+1}-1}\left(\frac{1}{2}-\lambda_{i}\right) T\left(e_{i}\right)\right\| \leq \operatorname{dim} F \max _{2^{m} \leq i<2^{m+1}}\left\|T\left(e_{i}\right)\right\|=\gamma_{m} \operatorname{dim} F<\varepsilon .
$$

Observe that for $I_{1}=\left\{i=2^{m}, \ldots, 2^{m+1}-1: \lambda_{i}=0\right\}$ and $I_{2}=\{i=$ $\left.2^{m}, \ldots, 2^{m+1}-1: \lambda_{i}=1\right\}$ the vectors $f_{j}=\sum_{i \in I_{j}} e_{i}, j=1,2$ are $\mathrm{MC}$ fragments of $e$ and by (3.1),

$$
\left\|T\left(f_{1}\right)-T\left(f_{2}\right)\right\|=\left\|\sum_{i=2^{m}}^{2^{m+1}-1}\left(1-2 \lambda_{i}\right) T\left(e_{i}\right)\right\|<\varepsilon
$$

Now we are ready to prove the main result of the section.

Proof of Theorem 3.2. We may consider $F$ as a subspace of some $l_{\infty}(D)$ space

$$
F \hookrightarrow F^{\star \star} \hookrightarrow l_{\infty}\left(B_{F^{\star}}\right)=l_{\infty}(D)=W .
$$

By the notation $\hookrightarrow$ we mean an isometric embedding. It is well known that if $H$ is a relatively compact subset of $l_{\infty}(D)$ for some infinite set $D$ and $\varepsilon>0$ then there exists a finite rank operator $S \in l_{\infty}(D)$ such that $\|x-S x\| \leq \varepsilon$ for every $x \in H$ [18, Lemma 10.25]. Fix any $e \in E$ and $\varepsilon>0$. Since $T$ is a $C$-compact operator, $K=\{T(g): g$ is a fragment of $f\}$ is relatively 
compact in $X$ and hence, in $W$. By the above, there exist a finite rank linear operator $S \in \mathcal{L}(W)$ such that $\|f-S f\| \leq \frac{\varepsilon}{4}$ for every $f \in K$. Then $R=S \circ T$ is an orthogonally additive laterally-norm continuous finite rank operator. By Lemma 3.7 there exist $M C$ fragments $e_{1}, e_{2}$ of $e$ such that $\left\|R\left(e_{1}\right)-R\left(e_{2}\right)\right\|<\frac{\varepsilon}{2}$. Thus,

$$
\begin{aligned}
& \left\|T\left(e_{1}\right)-T\left(e_{2}\right)\right\| \\
& =\left\|T\left(e_{1}\right)-T\left(e_{2}\right)+S\left(T\left(e_{1}\right)\right)-S\left(T\left(e_{2}\right)\right)-S\left(T\left(e_{1}\right)\right)+S\left(T\left(e_{2}\right)\right)\right\| \\
& =\left\|T\left(e_{1}\right)-T\left(e_{2}\right)+R\left(e_{1}\right)-R\left(e_{2}\right)-S\left(T\left(e_{1}\right)\right)+S\left(T\left(e_{2}\right)\right)\right\| \\
& \leq\left\|R\left(e_{1}\right)-R\left(e_{2}\right)\right\|+\left\|T\left(e_{1}\right)-S\left(T\left(e_{1}\right)\right)-\left(T\left(e_{2}\right)-S\left(T\left(e_{2}\right)\right)\right)\right\| \\
& <\frac{\varepsilon}{2}+\frac{\varepsilon}{2}=\varepsilon .
\end{aligned}
$$

\section{Domination Problem For abstract Uryson narrow operators}

In this section we consider a domination problem for the modulus of abstract Uryson operators. In the classical sense, the domination problem can be stated as follows. Let $E, F$ be vector lattices, $S, T: E \rightarrow F$ linear operators with $0 \leq S \leq T$. Let $\mathcal{P}$ be some property of linear operators $R: E \rightarrow F$, so that $\mathcal{P}(R)$ means that $R$ possesses $\mathcal{P}$. Does $\mathcal{P}(T)$ imply $\mathcal{P}(S)$ ? Another version: if $|S| \leq T$, then whether $\mathcal{P}(T)$ implies $\mathcal{P}(S)$ ? See [2] for a survey on the domination problem for "small" operators.

Let $E, F$ be vector lattices with $E$ atomless, $T: E \rightarrow F$ a linear operator, $E_{1}$ a vector sublattice of $E, x_{0} \in E_{1}$ and $\mathfrak{F}_{x_{0}} \subseteq E_{1}$. Suppose $S: E_{1} \rightarrow F$ is an abstract Uryson operator with $\left.S\right|_{\mathfrak{F}_{x_{0}}}=\left.T\right|_{\mathfrak{F}_{x_{0}}}$. Obviously, $S$ is locally narrow at $x_{0}$ if and only if $T$ is.

Theorem 4.1. Let $E, F$ be vector lattices, with $E$ atomless and $F$ an ideal of some order continuous Banach lattices. Then every abstract Uryson operator $T: E \rightarrow F$ is order narrow if and only if is $|T|$ is.

First we need the following lemma.

Lemma 4.2. Let $E$ be a vector lattice, $T: E \rightarrow L_{1}(\mu)$ an abstract Uryson operator, $\varepsilon>0, n \in \mathbb{N}, x, y_{k}, u_{k}, v_{k} \in E$ for $k=1, \ldots, n$ satisfy $y_{k}=u_{k} \sqcup v_{k}$,

$$
x=\bigsqcup_{i=1}^{n} y_{i} \text { and }\left\||T|(x)-\sum_{i=1}^{n}\left|T\left(y_{i}\right)\right|\right\|<\varepsilon .
$$

Then

$$
\sum_{i=1}^{n}\left(\left\||T|\left(u_{i}\right)-\left|T\left(u_{i}\right)\right|\right\|+\left\||T|\left(v_{i}\right)-\left|T\left(v_{i}\right)\right|\right\|\right)<\varepsilon .
$$


Proof of Lemma 4.2. Since $x=\bigsqcup_{k=1}^{n}\left(u_{k} \sqcup v_{k}\right)$, by (3) of Theorem 1.4 and the orthogonal additivity of $T$ and $|T|$, one has

$$
0 \leq|T|(x)-\sum_{k=1}^{n}\left(\left|T\left(u_{k}\right)\right|+\left|T\left(v_{k}\right)\right|\right) \leq|T|(x)-\sum_{k=1}^{n}\left|T\left(y_{k}\right)\right|
$$

and

$$
|T|(x)=\sum_{k=1}^{n}\left(|T|\left(u_{k}\right)+|T|\left(v_{k}\right)\right) .
$$

Since $|T|\left(u_{k}\right)-\left|T\left(u_{k}\right)\right|$ and $|T|\left(v_{k}\right)-\left|T\left(v_{k}\right)\right|$ are positive elements of $L_{1}(\mu)$ for all $k \in\{1, \ldots, n\}$, the sum of their norms equals the norm of their sum. Thus, taking into account (4.3) and (4.4), we obtain

$$
\begin{aligned}
& \sum_{k=1}^{n}\left(\left\||T|\left(u_{k}\right)-\left|T\left(u_{k}\right)\right|\right\|+\left\||T|\left(v_{k}\right)-\left|T\left(v_{k}\right)\right|\right\|\right) \\
& =\left\||T|(x)-\sum_{k=1}^{n}\left(\left|T\left(u_{k}\right)\right|+\left|T\left(v_{k}\right)\right|\right)\right\| \leq\left\||T|(x)-\sum_{k=1}^{n}\left|T\left(y_{k}\right)\right|\right\|<\varepsilon .
\end{aligned}
$$

Before the proof of Theorem 4.1, we make a comment on it. The general idea is the same as in the linear case: first we consider the case of $F=L_{1}(\mu)$, and then prove in the general case using this partial case. The proof of the first part has some minor adjustments. However, the proof of the second part is quite different. We cannot use the same idea, because in the nonlinear case the image $T\left(I_{x}\right)$ of an order ideal $I_{x}$ in $E$ generated by $x \in E$ under an abstract Uryson operator $T: E \rightarrow F$ need not be contained in the order ideal $I_{T(x)}$ in $F$ generated by $T(x)$.

The next lemma presents the main tool for the proof of Theorem 4.1

Lemma 4.3. Let $E, F$ be vector lattices, with $E$ atomless and $F$ an ideal of some order continuous Banach lattices and $x_{0} \in E$. Then every abstract Uryson operator $T: E \rightarrow F$ is locally order narrow at $x_{0}$ if and only if is $|T|$ is.

Proof of Lemma 4.3. First we prove the lemma for the case of $F=L_{1}(\mu)$. By Lemma 2.8, we consider local narrowness instead of the local order narrowness. Fix any $x_{0} \in E$ and assume first that $T$ is locally narrow at $x_{0}$. Fix any $x \in \mathcal{F}_{x_{0}}$ and $\varepsilon>0$. By (3) of Theorem 1.4 ,

$$
\left\{|T|(x)-\sum_{k=1}^{n}\left|T\left(y_{k}\right)\right|: \quad x=\bigsqcup_{k=1}^{n} y_{k} ; \quad n \in \mathbb{N}\right\} \downarrow 0 .
$$

Hence, by the order continuity of $L_{1}(\mu)$, we can choose $y_{1}, \ldots, y_{n} \in \mathcal{F}_{x}$ such that (4.1) holds. 
For each $k=1, \ldots, n$ we represent $y_{k}=u_{k} \sqcup v_{k}$ so that $u_{k}, v_{k} \in E$ and $\left\|T\left(u_{k}\right)-T\left(v_{k}\right)\right\|<\varepsilon / n$. Then putting $u=\bigsqcup_{k=1}^{k} u_{k}, v=\bigsqcup_{k=1}^{n} v_{k}$, we obtain $x=u \sqcup v$ and by Lemma 4.2

$$
\begin{aligned}
& \||T|(u)-|T|(w)\| \leq \sum_{k=1}^{n}\left\||T|\left(u_{k}\right)-|T|\left(v_{k}\right)\right\| \leq \\
& \sum_{k=1}^{n}\left\|\left|T\left(u_{k}\right)\right|-\left|T\left(v_{k}\right)\right|\right\|+\sum_{k=1}^{n}\left(\left\||T|\left(u_{k}\right)-\left|T\left(u_{k}\right)\right|\right\|+\left\||T|\left(v_{k}\right)-\left|T\left(v_{k}\right)\right|\right\|\right) \\
& <\sum_{k=1}^{n}\left\|T\left(u_{k}\right)-T\left(v_{k}\right)\right\|+\varepsilon<2 \varepsilon .
\end{aligned}
$$

By arbitrariness of $x \in \mathcal{F}_{x_{0}}$ and $\varepsilon>0,|T|$ is locally narrow at $x_{0}$.

Now let $|T|$ be locally narrow at $x_{0}, x \in \mathcal{F}_{x_{0}}, \varepsilon>0$. Like in the first part of the proof, we choose choose $y_{1}, \ldots, y_{n} \in \mathcal{F}_{x}$ so that (4.1) is satisfied. For each $k=1, \ldots, n$ we decompose $y_{k}=u_{k} \sqcup v_{k}$ so that $\left\||T|\left(u_{k}\right)-|T|\left(v_{k}\right)\right\|<\varepsilon / n$ and set $u=\bigsqcup_{k=1}^{k} u_{k}$ and $v=\bigsqcup_{k=1}^{n} v_{k}$.

Since $|a-b|+|a+b|=|a|+|b|+|| a|-| b||$ for all $a, b \in \mathbb{R}$, we have that for each $f, g \in L_{1}(\mu)$

$$
\|f-g\|=\||f|-|g|\|+\|f\|+\|g\|-\|f+g\| .
$$

Observe that since $x=\bigsqcup_{k=1}^{n}\left(u_{k} \sqcup v_{k}\right)$, by (3) of Theorem 1.4,

$$
\left\|\sum_{k=1}^{n}\left(\left|T\left(u_{k}\right)\right|+\left|T\left(v_{k}\right)\right|\right)\right\| \leq\||T|(x)\|,
$$

and by the triangle inequality,

$$
\begin{aligned}
& \left\|\left|T\left(u_{k}\right)\right|-\left|T\left(v_{k}\right)\right|\right\| \\
\leq & \left\||T|\left(u_{k}\right)-|T|\left(v_{k}\right)\right\|+\left\||T|\left(u_{k}\right)-\left|T\left(u_{k}\right)\right|\right\|+\left\||T|\left(v_{k}\right)-\left|T\left(v_{k}\right)\right|\right\|
\end{aligned}
$$

for all $k=1, \ldots, n$. Since the $L_{1}$-norm of a sum of positive elements equals the sum of their norms, using (4.5) for $f=T\left(u_{k}\right), g=T\left(v_{k}\right)$, (4.6) and 
(4.7), we obtain

$$
\begin{aligned}
& \|T(u)-T(v)\| \leq \sum_{k=1}^{n}\left\|T\left(u_{k}\right)-T\left(v_{k}\right)\right\| \\
& =\sum_{k=1}^{n}\left\|\left|T\left(u_{k}\right)\right|-\left|T\left(v_{k}\right)\right|\right\|+\left\|\sum_{k=1}^{n}\left(\left|T\left(u_{k}\right)\right|+\left|T\left(v_{k}\right)\right|\right)\right\|-\left\|\sum_{k=1}^{n}\left|T\left(y_{k}\right)\right|\right\| \\
& \leq \sum_{k=1}^{n}\left\||T|\left(u_{k}\right)-|T|\left(v_{k}\right)\right\|+\sum_{k=1}^{n}\left(\left\||T|\left(u_{k}\right)-\left|T\left(u_{k}\right)\right|\right\|\right. \\
& \left.+\left\||T|\left(v_{k}\right)-\left|T\left(v_{k}\right)\right|\right\|\right)+\||T|(x)\|-\left\|\sum_{k=1}^{n}\left|T\left(y_{k}\right)\right|\right\| \\
& \quad \underset{\text { by Lemma } 4.2}{<}{ }_{n} \frac{\varepsilon}{n}+\varepsilon+\left\||T|(x)-\sum_{k=1}^{n}\left|T\left(y_{k}\right)\right|\right\|<3 \varepsilon
\end{aligned}
$$

by the choose of $y_{1}, \ldots, y_{n} \in \mathcal{F}_{x}$. Thus, $T$ is locally narrow at $x_{0}$.

Now we consider the general case. Fix $x_{0} \in E$ and any $x \in \mathcal{F}_{x_{0}}$. For each $\pi \in \Pi_{x}$ we set $L_{\pi}=$ linear $\operatorname{span} \pi$. Note that $\pi_{1} \leq \pi_{2}$ in $\Pi_{x}$ implies $L_{\pi_{1}} \subseteq L_{\pi_{2}}$. In particular, $\left(L_{\pi}\right)_{\pi \in \Pi_{x}}$ is a net with respect to the inclusion. The following linear subspace is a sublattice of $E$

$$
E_{1}=\bigcup_{\pi \in \Pi_{x}} L_{\pi}=\text { linear } \operatorname{span}\left(\mathfrak{F}_{x}\right) .
$$

For each $\pi=\left(x_{i}\right)_{i=1}^{n} \in \Pi_{x}$ we define the linear operator $S_{\pi}: L_{\pi} \rightarrow F$ which extends the equality $S_{\pi} x_{i}=T\left(x_{i}\right)$ for $i=1, \ldots, n$ to $L_{\pi}$ by linearity. By orthogonal additivity of $T$, if $\pi_{1} \leq \pi_{2}$ in $\mathfrak{F}_{x}$ then $\left.S_{\pi_{2}}\right|_{L_{\pi_{1}}}=S_{\pi_{1}}$. Thus, we can define the following orthogonally additive operator $S: E_{1} \rightarrow F$ by

$$
S(x)=\lim _{\pi \in \Pi_{x}} S_{\pi}(x), x \in E_{1} .
$$

By the construction of $S$,

$$
\left.S\right|_{\mathfrak{F}_{x_{0}}}=\left.T\right|_{\mathfrak{F}_{x_{0}}} \text { and }\left.|S|\right|_{\mathfrak{F}_{x_{0}}}=\left.|T|\right|_{\mathfrak{F}_{x_{0}}}
$$

(the second equality follows from (3) of Theorem 1.4).

By (4.8), $T$ is locally order narrow at $x_{0}$ if and only if $S$ is order narrow. By Theorem 4.1 for linear operators (see [18, Theorem 10.26]), $S$ is order narrow if and only if $|S|$ is order narrow. Finally, by (4.8), $|S|$ is order narrow if and only if $|T|$ is locally order narrow at $x_{0}$.

Proof of Theorem 4.1. Let $T \in \mathcal{U}(E, F)$ be an order narrow operator. Then $T$ is locally order narrow at every $x \in E$. By Lemma 4.3, $|T|$ is locally order narrow at every $x_{0} \in E$ and therefore $|T|$ is order narrow. The converse assertion can be proved by the same arguments.

Remark that, to prove Theorem 4.1 for the general case, we have used the case of $F=L_{1}(\mu)$ for linear operators only. However, we could not use the 
corresponding theorem from [10] for linear operators, because it was proved under superfluous assumptions of Dedekind completeness of $E$ and order continuity of $T$.

\section{5. $\lambda$-NARROW ABSTRACT URYSON OPERATORS}

In this section, by analogy with the linear case, we consider $\lambda$-narrow abstract Uryson operators acting between vector lattices.

Let $E, F$ be vector lattices with $F$ Dedekind complete. For an abstract Uryson operator $T: E \rightarrow F$ we define a function $\lambda_{T}: E^{+} \rightarrow F^{+}$by setting

$$
\lambda_{T}(x)=\bigwedge_{\pi \in \Pi_{x}} \bigvee_{y \in \pi}|T(y)|
$$

for every $x \in E^{+}$. Since $T$ is order bounded and $F$ is Dedekind complete, the function $\lambda_{T}$ is well defined. We say that $\lambda_{T}$ is the Enflo-Starbird function of $T$.

Definition 5.1. Let $E, F$ be vector lattices with $F$ Dedekind complete and $E$ atomless. An abstract Uryson operator $T: E \rightarrow F$ is called $\lambda$-narrow if $\lambda_{T}(x)=0$ for every $x \in E$.

It is clear that $\lambda_{T}(x) \leq \lambda_{|T|}(x)$ for every abstract Uryson operator $T$ and every $x \in E^{+}$. The following lemma is an analogue of Lemma 2.8 for $\lambda$-narrow operators.

Lemma 5.2. Let $E, F, G$ be vector lattices such that, $E$ is atomless and $F$ is an ideal of $G$. An abstract Uryson operator $T: E \rightarrow F$ is $\lambda$-narrow if and only if $T: E \rightarrow G$ is $\lambda$-narrow.

Proof. Fix any $x \in E^{+}$and set $\lambda_{\pi}=\bigvee_{u \in \pi}|T u|$ for each $\pi \in \Pi_{x}$ of $x$. By (5.1),$\lambda_{T}(x)=\inf _{\pi \in \Pi_{x}} \lambda_{\pi}$. Since $F$ is an ideal of $G$, we have that $\inf _{\pi \in \Pi_{x}} \lambda_{\pi}=0$ in $F$ if and only if $\inf _{\pi \in \Pi_{x}} \lambda_{\pi}=0$ in $G$.

We also need the following known lemma.

Lemma 5.3 (10], [18], Lemma 7.50). Let $z_{1}, \ldots, z_{2 n} \in L_{1}^{+}(\mu), K=\sum_{i=1}^{2 n}\left\|z_{i}\right\|$, $z_{0}=\bigvee_{i=1}^{2 n} z_{i}$ and $\alpha=\left\|z_{0}\right\|$. Then there exists a permutation $\tau:\{1, \ldots, 2 n\} \rightarrow$ $\{1, \ldots, 2 n\}$ such that

$$
\left\|\sum_{i=1}^{2 n}(-1)^{i} z_{\tau(i)}\right\| \leq \sqrt{2 \alpha K} .
$$

Theorem 5.4. Let $E, F$ be vector lattices with $F$ Dedekind complete, such that $E$ is atomless and $F$ is an ideal of an order continuous Banach lattice $G$. Then a positive abstract Uryson operator $T: E \rightarrow F$ is $\lambda$-narrow if and only if $T$ is order narrow.

Proof. By Lemma 2.7, instead of the order narrowness we consider narrowness. Assume $T$ is narrow. We show that $T$ is $\lambda$-narrow. Fix any $x \in E$ and $\varepsilon>0$. It is enough to prove that there is $\pi=\left\{x_{i}: i=1, \ldots, 2^{m}\right\} \in \Pi_{x}$ such 
that $\left\|\bigvee_{i=1}^{2^{m}} T\left(x_{i}\right)\right\|<\varepsilon$. Choose $m$ so that $2^{-m}\|T(x)\|<\varepsilon / 2$. Using that $T$ is narrow and orthogonally additive, we construct a disjoint tree $\left(y_{k}\right)_{k=1}^{\infty}$ on $x$ so that $\left\|T\left(y_{2 k}\right)-T\left(y_{2 k+1}\right)\right\|<2^{-m-1} \varepsilon \stackrel{\text { def }}{=} \varepsilon_{1}$. Then we claim that

$$
\left\|T\left(y_{2^{n}+j}\right)-2^{-n} T(x)\right\|<\varepsilon_{1} \text { for all } n=1,2, \ldots j=0,1, \ldots, 2^{n}-1 .
$$

Indeed, for $n=1$ and $j=0,1$ this follows from the next observation

$$
\left\|T\left(y_{2+j}\right)-2^{-1} T(x)\right\|=\frac{1}{2}\left\|T\left(y_{2}\right)-T\left(y_{3}\right)\right\|<\varepsilon_{1} .
$$

Supposing the claim is true for a given $n$ and all $j=0,1, \ldots, 2^{n}-1$, we obtain for $n+1$ and all $j=0,1, \ldots, 2^{n}-1$

$$
\begin{aligned}
& \left\|T\left(y_{2^{n+1}+2 j}\right)-2^{-n-1} T(x)\right\|=\frac{1}{2}\left\|2 T\left(y_{2^{n+1}+2 j}\right)-2^{-n} T(x)\right\| \\
& \leq \frac{1}{2}\left\|2 T\left(y_{2^{n+1}+2 j}\right)-T\left(y_{2^{n}+i}\right)\right\|+\frac{1}{2}\left\|T\left(y_{2^{n}+i}\right)-2^{-n} T(x)\right\| \\
& <\frac{1}{2}\left\|T\left(y_{2^{n+1}+2 j}\right)-T\left(y_{2^{n+1}+2 j+1}\right)\right\|+\frac{\varepsilon_{1}}{2}<\frac{\varepsilon_{1}}{2}+\frac{\varepsilon_{1}}{2}=\varepsilon_{1} .
\end{aligned}
$$

Analogously, $\left\|T\left(y_{2^{n+1}+2 j+1}\right)-2^{-n-1} T(x)\right\|<\varepsilon_{1}$, and the claim is proved by induction.

Now set $x_{i}=y_{2^{m}+i-1}$ for $i=1, \ldots, 2^{m}$. Then $x=\bigsqcup_{i=1}^{2^{m}} x_{i}$ and, by (15.2), $\left\|T\left(x_{i}\right)-2^{-m} T(x)\right\|<\varepsilon_{1}$ for $i=1, \ldots, 2^{m}$. Hence,

$$
\frac{T(x)}{2^{m}}-\bigvee_{i=1}^{2^{m}} T\left(x_{i}\right) \leq \frac{T(x)}{2^{m}}-T\left(x_{1}\right) \leq \sum_{i=1}^{2^{m}}\left|\frac{T(x)}{2^{m}}-T\left(x_{i}\right)\right|
$$

(in the middle part of the previous inequality one can put any of $x_{k}$ instead of $x_{1}$ ). On the other hand,

$$
T\left(x_{k}\right)=\frac{T(x)}{2^{m}}+\left(T\left(x_{k}\right)-\frac{T(x)}{2^{m}}\right) \leq \frac{T(x)}{2^{m}}+\sum_{i=1}^{2^{m}}\left|\frac{T(x)}{2^{m}}-T\left(x_{i}\right)\right|
$$

for every $1 \leq k \leq 2^{m}$. Therefore

$$
\bigvee_{k=1}^{2^{m}} T\left(x_{k}\right) \leq \frac{T(x)}{2^{m}}+\sum_{i=1}^{2^{m}}\left|\frac{T(x)}{2^{m}}-T\left(x_{i}\right)\right|
$$

and hence

$$
\bigvee_{k=1}^{2^{m}} T\left(x_{k}\right)-\frac{T(x)}{2^{m}} \leq \sum_{i=1}^{2^{m}}\left|\frac{T(x)}{2^{m}}-T\left(x_{i}\right)\right|
$$


Then (5.3) and (5.4) imply

$$
\begin{aligned}
\left\|\bigvee_{i=1}^{2^{m}} T\left(x_{i}\right)-\frac{T(x)}{2^{m}}\right\| & \leq\left\|\sum_{i=1}^{2^{m}}\left|\frac{T(x)}{2^{m}}-T\left(x_{i}\right)\right|\right\| \\
& \leq \sum_{i=1}^{2^{m}}\left\|\frac{T(x)}{2^{m}}-T\left(x_{i}\right)\right\|<2^{m} \varepsilon_{1}=\frac{\varepsilon}{2} .
\end{aligned}
$$

Finally we obtain

$$
\left\|\bigvee_{i=1}^{2^{m}} T\left(x_{i}\right)\right\| \leq\left\|\bigvee_{i=1}^{2^{m}} T\left(x_{i}\right)-\frac{T(x)}{2^{m}}\right\|+\left\|\frac{T(x)}{2^{m}}\right\|<\frac{\varepsilon}{2}+\frac{\varepsilon}{2}=\varepsilon .
$$

Thus, $T$ is $\lambda$-narrow.

Now let $T$ be a $\lambda$-narrow operator. We prove that $T$ is narrow. Like in the proof of Theorem 4.1, we consider the case of $F=L_{1}(\mu)$, and the general case can be reduced to this one exactly like in Theorem 4.1). Fix any $x \in E$ and $\varepsilon>0$. It $T(x)=0$ then $T(y)=0$ for each $y \sqsubseteq x$ by positivity and orthogonal additivity of $T$, and there is nothing to prove. Assume $T(x) \neq 0$. By positivity of $T$, the net $\left(\bigvee_{y \in \pi} T(y)\right)_{\pi \in \Pi_{x}}$ is decreasing. Then we can write

$$
\lambda_{T}(x)=\lim _{\pi \in \Pi_{x}} \bigvee_{y \in \pi} T(y)=0 .
$$

Using the order continuous of $F$, we find $\pi=\left\{x_{i}: i=1, \ldots, 2 n\right\} \in \Pi_{x}$, so that

$$
\alpha=\left\|\bigvee_{i=1}^{2 n} T\left(x_{i}\right)\right\| \leq \frac{\varepsilon^{2}}{2\|T(x)\|} .
$$

Now we put $z_{i}=T\left(x_{i}\right)$ for $1 \leq i \leq 2 n$. Note that $K=\sum_{i=1}^{2 n}\left\|z_{i}\right\|=\|T(x)\|$. Choose by Lemma 5.3 a permutation $\tau:\{1, \ldots, 2 n\} \rightarrow\{1, \ldots, 2 n\}$ so that

$$
\left\|\sum_{i=1}^{2 n}(-1)^{i} z_{\tau(i)}\right\| \leq \sqrt{2 \alpha K} \leq \varepsilon
$$

Then for $y_{1}=\sum_{k=1}^{n} x_{\tau(2 k-1)}$ and $y_{2}=\sum_{k=1}^{n} x_{\tau(2 k)}$ we have $x=y_{1} \sqcup y_{2}$ and

$$
\left\|T\left(y_{1}\right)-T\left(y_{2}\right)\right\|=\left\|\sum_{i=1}^{2 n}(-1)^{i} z_{\tau(i)}\right\|<\varepsilon .
$$

\section{Pseudo narrow abstract Uryson operators}

Let $E, F$ be vector lattices. An abstract Uryson operator $T: E \rightarrow F$ is called disjointness preserving if $T(x) \perp T(y)$ for all $x, y \in E$ with $x \perp y$. As we will see later (see Theorem 7.3 ), the set of all disjointness preserving abstract Uryson operators is solid. In particular, an abstract Uryson operator $T$ : $E \rightarrow F$ is disjointness preserving if and only if $|T|$ is. 
Definition 6.1. Let $E, F$ be vector lattices. An abstract Uryson operator $T \in \mathcal{U}(E, F)$ is called pseudo-narrow if there is no disjointness preserving abstract Uryson operator $S \in \mathcal{U}(E, F)$ so that $0<S \leq|T|$.

Theorem 6.2. Let $E, F$ be vector lattices, $F$ Dedekind complete and $E$ atomless. If a positive abstract Uryson operator $T: E \rightarrow F$ is $\lambda$-narrow then $T$ is pseudo-narrow.

Proof. Suppose $T$ is not pseudo-narrow. Let $S \in \mathcal{U}(E, F)$ be a disjointness preserving operator, $0<S \leq T$ and $x \in E$, such that $S(x)>0$. Then for every representation $x=\bigsqcup_{i=1}^{n} x_{i}$ we have

$$
S(x)=\bigsqcup_{i=1}^{n} S\left(x_{i}\right)=\bigvee_{i=1}^{n} S\left(x_{i}\right) \leq \bigvee_{i=1}^{n} T\left(x_{i}\right)
$$

Hence $\lambda_{T}(x) \geq S(x)>0$ and $T$ is not $\lambda$-narrow.

The converse assertion will be proved under the additional assumption of the lateral continuity of $T$. We need the some preliminary lemmas. Recall that a vector lattice $E$ is said to have the projection property if every band of $E$ is a projection band.

Lemma 6.3. Let $E$ be an atomless vector lattice with the projection property, and $F$ a Dedekind complete vector lattice. Let $T \in \mathcal{U}^{+}(E, F)$ be a laterally continuous abstract Uryson operator, $e \in E$ and $f \in F^{+}$. Let $\varphi: \mathfrak{F}_{e} \rightarrow \mathfrak{F}_{f}$ be a Boolean homomorphism such that $\varphi(x) \leq T(x)$ for each $x \in \mathfrak{F}_{e}$. Then there exists a disjointness preserving abstract Uryson operator $S \in \mathcal{U}^{+}(E, F)$ such that $S \leq T$ and $S(x)=\varphi(x)$ for each $x \in \mathfrak{F}_{e}$.

Proof. For each $\pi=\left(x_{i}\right)_{i=1}^{n} \in \Pi_{e}$ we set $L_{\pi}=\operatorname{span}\left\{x_{i}: 1 \leq i \leq n\right\}$. We note that $\pi_{1} \leq \pi_{2}$ in $\Pi_{e}$ implies $L_{\pi_{1}} \subseteq L_{\pi_{2}}$. In particular, $\left(L_{\pi}\right)_{\pi \in \Pi_{e}}$ is an increasing net with respect to the inclusion. The following linear subspace is a sublattice of $E$

$$
G=\bigcup_{\pi \in \Pi_{e}} L_{\pi}=\operatorname{span} \mathfrak{F}_{e}
$$

(in other words, $G$ is the set of all $e$-step functions). For each $\pi=\left(x_{i}\right)_{i=1}^{n} \in$ $\Pi_{e}$ we define the orthogonally additive operator $S_{\pi}: L_{\pi} \rightarrow F$ which extends the equality $S_{\pi}\left(x_{i}\right)=\varphi\left(x_{i}\right)$ for $i=1, \ldots, n$ to $L_{\pi}$ by the following rule

$$
S_{\pi}\left(\sum_{i=1}^{n} \lambda_{i} x_{i}\right)=\sum_{i=1}^{n}\left|\lambda_{i}\right| S_{\pi}\left(x_{i}\right) .
$$

Observe that, if $y \in L_{\pi}$ with $|y| \leq e$ then $y=\sum_{i=1}^{n} \lambda_{i} x_{i}$ with $\left|\lambda_{i}\right| \leq 1$ for all $i$. Hence by (6.1),

$$
S_{\pi}(y) \leq \sum_{i=1}^{n} S_{\pi}\left(x_{i}\right)=\sum_{i=1}^{n} \varphi\left(x_{i}\right)=T(e) .
$$


Since $\varphi$ is a Boolean homomorphism on $\mathfrak{F}_{e}$, we have that if $\pi_{1} \leq \pi_{2}$ in $\mathfrak{F}_{e}$ then $\left.S_{\pi_{2}}\right|_{L_{\pi_{1}}}=S_{\pi_{1}}$. Thus, we can define the following orthogonally additive operator $S_{1}: G \rightarrow F$

$$
S_{1}(x)=\lim _{\pi \in \Pi_{e}} S_{\pi}(x), x \in G .
$$

Moreover, since $\varphi$ is a Boolean homomorphism, $S_{1}$ is a disjointness preserving operator.

Assume now that $m \in \mathbb{N}$ and $y \in E$ with $|y| \leq m e$. Then $m^{-1} y \leq e$ and for each $\pi \in \Pi_{e}$ by (6.2) and (6.1), $m^{-1} S_{\pi}(y)=S_{\pi}\left(m^{-1} y\right) \leq T(e)$, that is, $S_{\pi}(y) \leq m T(e)$. By arbitrariness of $\pi \in \Pi_{e}$ and (6.3), we obtain

$$
(\forall m \in \mathbb{N})(\forall y \in G)\left(|y| \leq m e \Rightarrow S_{1}(y) \leq m T(e)\right) .
$$

Let $I_{e}=\{x \in E:(\exists m \in \mathbb{N})(|x| \leq m e)\}$ be the order ideal generated by $e$. Fix any $x \in I_{e}$. Then $|x| \leq m e$ for some $m \in \mathbb{N}$. Then for any $y \in G$ with $|y| \leq|x|$ we have $|y| \leq m e$, and $S_{1}(y) \leq m T(e)$ by 6.4). Thus, the set $\left\{S_{1} y:|y| \leq|x|, y \in G\right\}$ is order bounded in $F$ by $m T(e)$. Since $F$ is Dedekind complete, there exists

$$
S_{2}(x)=\sup \left\{S_{1}(y):|y| \leq|x|, y \in G\right\}
$$

with $S_{2}(x) \leq m T(e)$. Moreover, if $A \subseteq I_{e}$ is an order bounded set by me then $S_{2}(y) \leq m T(e)$ for all $y \in A$.

We show that formula (6.5) defines a positive abstract Uryson operator $S_{2}: I_{e} \rightarrow F$. By the above, $S_{2}$ is an order bounded map. Since $S_{1} \geq 0$, we have that $S_{2}(x) \geq 0$ for all $x \in I_{e}$. So, it remains to show the orthogonal additivity of $S_{2}$. Let $x_{1}, x_{2} \in I_{e}$ and $x_{1} \perp x_{2}$. Then

$$
S_{2}\left(x_{1}+x_{2}\right)=\sup \left\{S_{1}(y):|y| \leq\left|x_{1}+x_{2}\right|=\left|x_{1}\right|+\left|x_{2}\right|, y \in G\right\} .
$$

Let $y \in G$ be any element with $|y| \leq\left|x_{1}\right|+\left|x_{2}\right|$. By the Riesz decomposition property [1, Theorem 1.15], there exist $y_{1}, y_{2} \in G$ so that $y=y_{1}+y_{2}$ and $\left|y_{i}\right| \leq\left|x_{i}\right|$ for $i \in\{1,2\}$. In particular, $y_{1} \perp y_{2}$. Then

$$
S_{1}(y)=S_{1}\left(y_{1}+y_{2}\right)=S_{1}\left(y_{1}\right)+S_{1}\left(y_{2}\right) \leq S_{2}\left(x_{1}\right)+S_{2}\left(x_{2}\right) .
$$

Passing to the supremum, we obtain $S_{2}\left(x_{1}+x_{2}\right) \leq S_{2}\left(x_{1}\right)+S_{2}\left(x_{2}\right)$.

On the other hand, if $y_{1}, y_{2} \in G$ satisfy $\left|y_{i}\right| \leq\left|x_{i}\right|$ for $i \in\{1,2\}$ then $y_{1} \perp y_{2}$ and hence

$$
S_{1}\left(y_{1}\right)+S_{1}\left(y_{2}\right)=S_{1}\left(y_{1}+y_{2}\right) \leq S_{2}\left(x_{1}+x_{2}\right) .
$$

Passing to the supremum first over $y_{1}$ and then over $y_{2}$, we get $S_{2}\left(x_{1}\right)+$ $S_{2}\left(x_{2}\right) \leq S_{2}\left(x_{1}+x_{2}\right)$. Thus, $S_{2}: I_{e} \rightarrow F$ is a positive abstract Uryson operator.

Let $\left.T\right|_{I_{e}}$ be the restriction of $T$ to the sublattice $I_{e}$. Set $S_{3}=\left.T\right|_{I_{e}} \wedge$ $S_{2}$. Using the fact that the set of all laterally continuous abstract Uryson operators from the vector lattice $I_{e}$ to the Dedekind complete vector lattice $F$ is a band in $\mathcal{U}(E, F)$ [12, Proposition 3.8], we have that $S_{3}: I_{e} \rightarrow F$ is a positive laterally continuous abstract Uryson operator. 
Denote by $B_{e}$ the band in $E$ generated by $e$. Now we extend $S_{3}$ from $I_{e}$ to a positive laterally continuous abstract Uryson operator $S_{4}: B_{e} \rightarrow F$. For any $x \in B_{e}$ we set

$$
S_{4}(x)=\sup \left\{S_{3}(y): y \sqsubseteq x, y \in I_{e}\right\} .
$$

The supremum exists because $F$ is Dedekind complete and $S_{3}(y) \leq T(y) \leq$ $T(x)$ for each $y \sqsubseteq x, y \in I_{e}$ by the inequality $S_{3} \leq\left. T\right|_{I_{e}}$. By the above, $T_{4}(x)=T_{3}(x)$ for all $x \in I_{e}$ and $S_{4}(x) \leq T(x)$ for all $x \in B_{e}$.

Show that $S_{4}$ is orthogonally additive. Let $x_{1}, x_{2} \in B_{e}$ and $x_{1} \perp x_{2}$. Then

$$
S_{4}\left(x_{1}+x_{2}\right)=\sup \left\{S_{3}(y): y \sqsubseteq x_{1}+x_{2}, y \in I_{e}\right\} .
$$

Let $y \in I_{e}$ satisfy $y \sqsubseteq x_{1}+x_{2}$. Since $I_{e}$ is an ideal in $E$, we have that $y_{i}=\left(y^{+} \wedge x_{i}^{+}\right)-\left(y^{-} \wedge x_{i}^{-}\right) \in I_{e}, i \in\{1,2\}$. Observe that $y_{i} \sqsubseteq x_{i}$ and $y_{1} \perp y_{2}$, hence,

$$
S_{3}(y)=S_{3}\left(y_{1}\right)+S_{3}\left(y_{2}\right) \leq S_{4}\left(x_{1}\right)+S_{4}\left(x_{2}\right) .
$$

Passing to the supremum, we obtain $S_{4}\left(x_{1}+x_{2}\right) \leq S_{4}\left(x_{1}\right)+S_{4}\left(x_{2}\right)$.

On the other hand, if $y_{1}, y_{2} \in I_{e}$ satisfy $y_{i} \sqsubseteq x_{i}$ for $i \in\{1,2\}$ then $y_{1} \perp y_{2}$ and hence

$$
S_{3}\left(y_{1}\right)+S_{3}\left(y_{2}\right)=S_{3}\left(y_{1}+y_{2}\right) \leq S_{4}\left(x_{1}+x_{2}\right) .
$$

Passing to the supremum firstly over $y_{1}$ and secondly over $y_{2}$, we get $S_{4}\left(x_{1}\right)+$ $S_{4}\left(x_{2}\right) \leq S_{4}\left(x_{1}+x_{2}\right)$. Thus, $S_{4}: B_{e} \rightarrow F$ is a positive abstract Uryson operator.

Now we show that $S_{4}$ is laterally continuous. Observe that, if $T_{1}: E_{1} \rightarrow$ $F_{1}$ is a positive abstract Uryson operator then the lateral continuity of $T_{1}$ at $z \in E_{1}$ is equivalent to the following property: if $\left(z_{\alpha}\right)$ is a $\sqsubseteq$-increasing net of fragments of $z$ with $z_{\alpha} \stackrel{\mathrm{o}}{\longrightarrow} z$ then $T_{1}(z) \leq \sup _{\alpha} T_{1}\left(z_{\alpha}\right)$. Indeed, the inequality $T_{1}(z) \geq \sup _{\alpha} T_{1}\left(z_{\alpha}\right)$ is also true because $T_{1}\left(z_{\alpha}\right) \leq T_{1}(z)$ for all indices $\alpha$, and $T_{1}\left(z_{\alpha}\right) \stackrel{\mathrm{o}}{\longrightarrow} \sup _{\alpha} T_{1}\left(z_{\alpha}\right)$ because the net $T_{1}\left(z_{\alpha}\right)$ increases.

So, let $x \in B_{e}$, let $\left(x_{\alpha}\right)$ be a net, $x_{\alpha} \sqsubseteq x_{\beta} \sqsubseteq x$ for $\alpha<\beta$ and $x_{\alpha} \stackrel{\circ}{\longrightarrow} x$. Fix any $y \in I_{e}$ with $y \sqsubseteq x$ and set $y_{\alpha}=\left(y^{+} \wedge x_{\alpha}^{+}\right)-\left(y^{-} \wedge x_{\alpha}^{-}\right)$. Then $y_{\alpha} \sqsubseteq x_{\alpha}, y_{\alpha} \sqsubseteq y$ for all $\alpha, y_{\alpha} \stackrel{\circ}{\longrightarrow} y$ and $y_{\alpha} \in I_{e}$. By the lateral continuity of $S_{3}$,

$$
S_{3}(y)=\sup _{\alpha} S_{3}\left(y_{\alpha}\right) \leq \sup _{\alpha} \sup \left\{S_{3}(z): z \sqsubseteq x_{\alpha}, z \in I_{e}\right\}=\sup _{\alpha} S_{4}\left(x_{\alpha}\right) .
$$

By arbitrariness of $y$,

$$
S_{4}(x)=\sup \left\{S_{3}(y): y \sqsubseteq x, y \in I_{e}\right\} \leq \sup _{\alpha} S_{4}\left(x_{\alpha}\right),
$$

and the lateral continuity of $S_{4}$ is proved.

Finally, we define an abstract Uryson operator $S: E \rightarrow F$ by $S=S_{4} \circ P_{e}$ where $P_{e}$ is the band projection of $E$ onto $B_{e}$.

Show that $S$ is a disjointness preserving operator. Assume, on the contrary, that $w=S x \wedge S y>0$ for some $x, y \in E$ with $x \perp y$. Set $u=P_{e} x$, $v=P_{e} y$. Then $u \perp v$ and $S_{4}(u) \wedge S_{4}(v)=w>0$. Using (6.6), we find $u_{1} \sqsubseteq u$ and $v_{1} \sqsubseteq v$ so that $u_{1}, v_{1} \in I_{e}$ and $w_{1}=S_{3}\left(u_{1}\right) \wedge S_{3}\left(v_{1}\right)>0$. Since 
$S_{2} \geq S_{3}$, we obtain $w_{2}=S_{2}\left(u_{1}\right) \wedge S_{2}\left(v_{1}\right) \geq w_{1}>0$. By (6.5), there are $u_{2}, v_{2} \in G$ such that $\left|u_{2}\right| \leq\left|u_{1},\right| v_{2}|\leq| v_{1}$ and $w_{3}=S_{1}\left(u_{2}\right) \wedge S_{1}\left(v_{2}\right)>0$. Since $\left|u_{2}\right| \leq\left|u_{1}\right| \leq|u|$ and analogously $\left|v_{2}\right| \leq|v|$, one has that $u_{2} \perp v_{2}$. This is impossible because $S_{1}$ is a disjointness preserving operator.

It remains to observe that $S(x)=S_{4}\left(P_{e} x\right) \leq T\left(P_{e} x\right) \leq T(x)$ for all $x \in E$.

\section{Pseudo-embeddings and a generalization of Rosenthal's DECOMPOSITION THEOREM TO ABSTRACT URYSON OPERATORS}

Let $E$ be a vector lattice. For an arbitrary index set $J$ a series $\sum_{j \in J} x_{j}$ of elements $x_{j} \in E$ is called order convergent and the family $\left(x_{j}\right)_{j \in J}$ is called order summable if the net $\left(y_{s}\right)_{s \in J<\omega}, y_{s}=\sum_{j \in s} x_{j}$ order converges to some $y_{0} \in E$, where $J<\omega$ is the net of all finite subsets $s \subseteq J$ ordered by inclusion. In this case $y_{0}$ is called the order sum of the series $\sum_{j \in J} x_{j}$ and we write $y_{0}=\sum_{j \in J} x_{j}$. A series $\sum_{j \in J} x_{j}$ is called absolutely order convergent and the family $\left(x_{j}\right)_{j \in J}$ is called absolutely order summable if the series $\sum_{j \in J}\left|x_{j}\right|$ order converges.

Let $A$ be a solid subset of a vector lattice $E$, that is, for every $x \in E$ and $y \in A$ the inequality $|x| \leq|y|$ implies $x \in A$. We denote by $\operatorname{Abs}(A)$ the set of all sums of absolutely order convergent series $\sum_{j \in J} x_{j}$ of elements $x_{j} \in A$. For any subset $B \subseteq E$ by $\operatorname{Band}(A)$ we denote the least band in $E$ containing $A$.

Lemma 7.1 (Theorem 1.25, [18]). Let $A$ be a solid subset of a Dedekind complete vector lattice $E$. Then

(i) $A^{d}=\{x \in E:$ for all $y \in A, \quad 0 \leq y \leq|x|$ implies $y=0\}$;

(ii) $\operatorname{Band}(A)=\operatorname{Abs}(A)$.

In particular, $E=\operatorname{Abs}(A) \oplus A^{d}$ is a decomposition into mutually complemented bands.

Let $E$ and $F$ be vector lattices. By $\mathcal{U}_{d p o}(E, F)$ we denote the set of all disjointness preserving abstract Uryson operators $T: E \rightarrow F$.

Definition 7.2. Let $E, F$ be vector lattices with $F$ Dedekind complete. An operator $T \in \mathcal{U}(E, F)$ is called pseudo embedding if there exists an absolutely order summable family $\left(T_{j}\right)_{j \in J}$ in $\mathcal{U}_{d p o}(E, F)$ such that $T=\sum_{j \in J} T_{j}$.

The name of "pseudo embedding" is due to Rosenthal's theorem concerning operators on $L_{1}$ which asserts that a non-zero operator is a pseudo embedding if and only if it is a near isometric embedding when restricted to a suitable $L_{1}(A)$-subspace [18, Theorem 7.39].

The set of all pseudo embeddings from $\mathcal{U}(E, F)$ will be denoted $\mathcal{U}_{p e}(E, F)$. Thus, $\mathcal{U}_{p e}(E, F)=\operatorname{Abs}\left(\mathcal{U}_{d p o}(E, F)\right)$ by definition. The set of all pseudo narrow operators $T \in \mathcal{U}(E, F)$ will be denoted $\mathcal{U}_{p n}(E, F)$.

Theorem 7.3. Let $E, F$ be vector lattices with $F$ Dedekind complete. Then 
(i) $\mathcal{U}_{\text {dpo }}(E, F)$ is solid in $\mathcal{U}(E, F)$;

(ii) Band $\left(\mathcal{U}_{\text {dpo }}(E, F)\right)=\mathcal{U}_{p e}(E, F)$;

(iii) $\mathcal{U}_{d p o}(E, F)^{d}=\mathcal{U}_{p n}(E, F)$;

(iv) $\mathcal{U}_{p e}(E, F)$ and $\mathcal{U}_{p n}(E, F)$ are mutually complemented bands, hence $\mathcal{U}(E, F)=\mathcal{U}_{p e}(E, F) \oplus \mathcal{U}_{p n}(E, F)$.

Proof. By Lemma 7.1, it is enough to prove (i). Suppose $S \in \mathcal{U}(E, F)$, $T \in \mathcal{U}_{d p o}(E, F)$ and $|S| \leq|T|$. Our goal is to prove that $S \in \mathcal{U}_{d p o}(E, F)$. First we prove that $|T| \in \mathcal{U}_{\text {dpo }}(E, F)$. Let $x, y \in E$ and $x \perp y$. By (1) of Theorem 1.3 ,

$$
\begin{aligned}
& |T|(x)=\sup \{T(u)-T(v): x=u \sqcup v\} ; \\
& |T|(y)=\sup \{T(w)-T(z): y=w \sqcup z\} .
\end{aligned}
$$

Let $x=u \sqcup v$ and $y=w \sqcup z$. Since $T$ is disjointness preserving, $\mid T(u)-$ $T(v)|=| T(u)+T(v)|=| T(u+v)|=| T(x) \mid$. Analogously, $|T(w)-T(z)|=$ $|T(y)|$. Hence,

$$
|T(u)-T(v)| \wedge|T(w)-T(z)|=|T(x)| \wedge|T(y)|=0 .
$$

Passing to the supremum, we obtain $|T|(x) \perp|T|(y)$. Thus, $|T| \in \mathcal{U}_{d p o}(E, F)$.

Now we prove that $S \in L_{d p o}(E, F)$. Let $x_{1}, x_{2} \in E, x \perp y$. Since $\left|S x_{i}\right| \leq$ $|S|\left|x_{i}\right| \leq|T|\left|x_{i}\right|, i=1,2$, we obtain that

$$
0 \leq\left|S x_{1}\right| \wedge\left|S x_{2}\right| \leq|T|\left|x_{1}\right| \wedge|T|\left|x_{2}\right|=0
$$

Thus, $S x_{1} \perp S x_{2}$.

By [12, Proposition 3.8], the set $\mathcal{U}^{l c}(E, F)$ of all laterally continuous abstract Uryson operators from $E$ to $F$ is a band in $\mathcal{U}(E, F)$. Since the intersection of bands is a band, we obtain the following version of Theorem 7.3 for order continuous operators.

Corollary 7.4. Let $E, F$ be vector lattices with $F$ Dedekind complete. Then

$(i)$ the set $\mathcal{U}_{d p o}^{l c}(E, F)$ of all disjointness preserving laterally continuous operators is solid in $\mathcal{U}^{l c}(E, F)$;

(ii) Band $\left(\mathcal{U}_{d p o}^{l c}(E, F)\right)=\mathcal{U}_{p e}^{l c}(E, F)$;

(iii) $\mathcal{U}_{d p o}^{l c}(E, F)^{d}=\mathcal{U}_{p n}^{l c}(E, F)$;

(iv) the sets $\mathcal{U}_{p e}^{l c}(E, F)$ and $\mathcal{U}_{p n}^{l c}(E, F)$ are mutually complemented bands, hence $\mathcal{U}^{l c}(E, F)=\mathcal{U}_{p e}^{l c}(E, F) \oplus \mathcal{U}_{p n}^{l c}(E, F)$.

Here, $\mathcal{U}_{d p o}^{l c}(E, F), \mathcal{U}_{p e}^{l c}(E, F)$ and $\mathcal{U}_{p n}^{l c}(E, F)$ denote the corresponding intersections of $\mathcal{U}_{\text {dpo }}(E, F), \mathcal{U}_{p e}(E, F)$ and $\mathcal{U}_{p n}(E, F)$ with $\mathcal{U}^{l c}(E, F)$.

\section{BOOLEAN MAPS}

We need some information about Boolean maps and homomorphisms.

Definition 8.1. Let $A, B$ be lattices (not necessarily vector spaces). A map $\psi: A \rightarrow B$ is said to be 
1) $\vee$-preserving if $\psi(x \vee y)=\psi(x) \vee \psi(y)$ for all $x, y \in A$.

2) ^-preserving if $\psi(x \wedge y)=\psi(x) \wedge \psi(y)$ for all $x, y \in A$.

3) a lattice homomorphism provided it is both $\vee$-preserving and $\wedge$ preserving.

If, moreover, $A$ and $B$ are Boolean algebras then in each of the above definitions we additionally claim that $\psi\left(\mathbf{0}_{A}\right)=\mathbf{0}_{B}$ and $\psi\left(\mathbf{1}_{A}\right)=\mathbf{1}_{B}$. In this case we insert the word "Boolean" (a Boolean $\vee$-preserving map; a Boolean $\wedge$-preserving map; a Boolean homomorphism).

We need the following two known facts.

Theorem 8.2 (Monteiro's theorem [18], Theorem 10.33). Let A, B be Boolean algebras with $B$ Dedekind complete and $\varphi: A \rightarrow B$ be a Boolean $\vee$-preserving map. Then every Boolean homomorphism $\psi_{0}: A_{0} \rightarrow B$ of a Boolean subalgebra $A_{0} \subseteq A$ with $\psi_{0}(x) \leq \varphi(x)$ for each $x \in A_{0}$ can be extended to a Boolean homomorphism $\psi: A \rightarrow B$ with $\psi(x) \leq \varphi(x)$ for each $x \in A$.

Recall that for the band projection $P_{e} x$ of an element $x \in E$ to the band generated by $e \in E^{+}$we have the formula $P_{e} x=\bigvee_{n=1}^{\infty}(x \wedge n e) \in \mathfrak{F}_{x}$ [1, Theorem 3.13].

Lemma 8.3 ([10], [18], Lemma 10.38). Let $F$ be a Dedekind complete vector lattice, $f \in F^{+}$. Then the formula

$$
\mathbf{1}_{f}(y)=f-P_{(f-y)}+f
$$

defines a lattice homomorphism $\mathbf{1}_{f}: F^{+} \rightarrow \mathfrak{F}_{f}$ such that $\mathbf{1}_{f}(y) \leq y$ for all $y \in F_{+}, \mathbf{1}_{f}(0)=0$ and $\mathbf{1}_{f}(f)=f$.

Now we are ready to prove the next auxiliary statement.

Lemma 8.4. Let $E, F$ be vector lattices with $F$ Dedekind complete. Let $T: E \rightarrow F$ be an abstract Uryson operator and $e \in E$ be such that $f=$ $\lambda_{T}(e)>0$. Then the formula $\varphi(x)=\mathbf{1}_{f}\left(\lambda_{T}(x)\right)$, where $\mathbf{1}_{f}$ is the map defined by (8.1), defines a Boolean $\vee$-preserving map $\varphi: \mathfrak{F}_{e} \rightarrow \mathfrak{F}_{f}$ such that $\varphi(x) \leq|T|(x)$ for all $x \in \mathfrak{F}_{e}$.

Proof. By Lemma 8.3, $\varphi(0)=\mathbf{1}_{f}\left(\lambda_{T}(0)\right)=\mathbf{1}_{f}(0)=0$ and $\varphi(e)=\mathbf{1}_{f}\left(\lambda_{T}(e)\right)=$ $\mathbf{1}_{f}(f)=f$. Fix any $x \in \mathfrak{F}_{e}$. Observe that, for any $\pi \in \Pi_{x}$ and any $u \in \pi$ one has $|T|(u) \leq|T|(x)$. Hence, $\bigvee_{u \in \pi}|T|(u) \leq|T|(x)$. Then we may write

$$
\begin{aligned}
\varphi(x) & =\mathbf{1}_{f}\left(\lambda_{T}(x)\right) \stackrel{\text { by Lemma }}{\leq} \lambda_{T}(x)=\bigwedge_{\pi \in \Pi_{x}} \bigvee_{u \in \pi}|T(u)| \\
& \leq \bigwedge_{\pi \in \Pi_{x}} \bigvee_{u \in \pi}|T|(u) \leq \bigwedge_{\pi \in \Pi_{x}}|T|(x)=|T|(x) .
\end{aligned}
$$


Now we prove that $\varphi$ is a $\bigvee$-preserving map. Show firstly that $\varphi(x \sqcup y)=$ $\varphi(x) \vee \varphi(y)$. Let $x, y \in \mathfrak{F}_{e}$ and $x \perp y$. Then we have

$$
\begin{aligned}
\lambda_{T}(x \sqcup y) & =\lim _{\pi \in \Pi_{x} \sqcup y} \bigwedge_{\pi^{\prime} \geq \pi} \bigvee_{u \in \pi^{\prime}}|T(u)|=\lim _{\Pi_{x} \sqcup y \ni \pi \geq\{x, y\}} \bigwedge_{\pi^{\prime} \geq \pi} \bigvee_{u \in \pi^{\prime}}|T(u)| \\
& =\lim _{\pi \in \Pi_{x}, \tau \in \Pi_{y}} \bigwedge_{\pi^{\prime} \geq \pi, \tau^{\prime} \geq \tau} \bigvee_{u \in \pi^{\prime} \cup \tau^{\prime}}|T(u)| \\
& =\lim _{\pi \in \Pi_{x}, \tau \in \Pi_{y}} \bigwedge_{\pi^{\prime} \geq \pi, \tau^{\prime} \geq \tau}\left(\bigvee_{u \in \pi^{\prime}}|T(u)| \vee \bigvee_{v \in \tau^{\prime}}|T(v)|\right) \\
& \left.=\lim _{\pi \in \Pi_{x}, \tau \in \Pi_{y}}\left(\bigwedge_{\pi^{\prime} \geq \pi} \bigvee_{u \in \pi^{\prime}}|T u|\right) \vee\left(\bigwedge_{\tau^{\prime} \geq \tau} \bigvee_{v \in \tau^{\prime}}|T v|\right)\right) \\
& =\lim _{\pi \in \Pi_{x}} \bigwedge_{\pi^{\prime} \geq \pi} \bigvee_{u \in \pi^{\prime}}|T u| \vee \lim _{\tau \in \Pi_{y}} \bigwedge_{\tau^{\prime} \geq \tau} \bigvee_{v \in \tau^{\prime}}|T v| \\
& =\bigwedge_{\pi \in \Pi_{x}} \bigvee_{u \in \pi}|T u| \vee \bigwedge_{\tau \in \Pi_{y}} \bigvee_{v \in \tau}|T v|=\lambda_{T}(x) \vee \lambda_{T}(y) .
\end{aligned}
$$

Hence, $\varphi(x \sqcup y)=\mathbf{1}_{e}\left(\lambda_{T}(x \sqcup y)\right)=\mathbf{1}_{e}\left(\lambda_{T}(x) \vee \lambda_{T}(y)\right)$

$$
=\mathbf{1}_{e}\left(\lambda_{T}(x)\right) \vee \mathbf{1}_{e}\left(\lambda_{T}(y)\right)=\varphi(x) \vee \varphi(y) .
$$

If $x, y \in \mathfrak{F}_{e}$ with $x \leq y$ then

$$
\varphi(y)=\varphi((y-x) \sqcup(x))=\varphi(y-x) \vee \varphi(x) \geq \varphi(x) .
$$

Therefore $\varphi(x \vee y) \geq \varphi(x) \vee \varphi(y)$ for all $x, y \in \mathfrak{F}_{e}$.

On the other hand, for all $x, y \in \mathfrak{F}_{e}$ one has

$$
\varphi(x \vee y)=\varphi((x-x \wedge y) \sqcup y)=\varphi(x-x \wedge y) \vee \varphi(y) \leq \varphi(x) \vee \varphi(y) .
$$

Thus $\varphi(x \vee y)=\varphi(x) \vee \varphi(y)$ for every $x, y \in \mathfrak{F}_{e}$.

\section{The MAin Results}

Theorem 9.1. Let $E$ be an atomless vector lattice with the projection property, $F$ a Dedekind complete vector lattice. Then a positive laterally continuous abstract Uryson operator $T: E \rightarrow F$ is $\lambda$-narrow if and only if is pseudo-narrow.

Proof. By Theorem 6.2 we only need to prove that a pseudo-narrow operator is $\lambda$-narrow. Suppose that $T$ is not $\lambda$-narrow and $e \in E$ is such that $f=$ $\lambda_{T}(e)>0$. By Lemma 8.4 we construct a Boolean $\vee$-preserving map $\varphi$ : $\mathfrak{F}_{e} \rightarrow \mathfrak{F}_{f}$ with the corresponding properties. Let $A=\mathfrak{F}_{e}, B=\mathfrak{F}_{f}, A_{0}=$ $\{0, e\}, \psi_{0}: A_{0} \rightarrow B$ be the trivial Boolean homomorphism (i.e. $\psi_{0}(0)=0$ and $\left.\psi_{0}(e)=f\right)$. Evidently, $\psi_{0}(x) \leq \varphi(x)$ for every $x \in A_{0}$. By Theorem 8.2 , there is a Boolean homomorphism $\psi: \mathfrak{F}_{e} \rightarrow \mathfrak{F}_{f}$ such that $\psi$ extends $\psi_{0}$ with $\psi(x) \leq \varphi(x)$ for all $x \in \mathfrak{F}_{e}$. By the choice of $\varphi$, one has that $\varphi(x) \leq T(x)$ for all $x \in \mathfrak{F}_{e}$. Thus, $\psi(x) \leq T(x)$ for all $x \in \mathfrak{F}_{e}$. By Lemma 6.3, there exists a disjointness preserving abstract Uryson operator $S: E \rightarrow F$ such 
that $0 \leq S \leq T$ and $S(x)=\psi(x)$ for all $x \in \mathfrak{F}_{e}$. In particular, $S(e)=$ $\psi(e)=\psi_{0}(e)=f>0$ and hence $S>0$. This means that $T$ is not pseudonarrow.

As a consequence of the above results, we obtain the following theorem.

Theorem 9.2. Let $E, F$ be vector lattices such that $E$ is atomless and has the projection property, $F$ an ideal of some order continuous Banach lattice. Then

(1) Every laterally continuous abstract Uryson operator $T: E \rightarrow F$ is order narrow if and only if it is pseudo-narrow.

(2) The set $\mathcal{U}_{o n}^{l c}(E, F)$ of all order narrow laterally continuous abstract Uryson operators is a band in the Dedekind complete vector lattice $\mathcal{U}^{l c}(E, F)$ of all laterally continuous abstract Uryson operator from $E$ to $F$. Moreover, the orthogonal complement to $\mathcal{U}_{o n}^{l c}(E, F)$ equals the band generated by all disjointness preserving laterally continuous abstract Uryson operators from $E$ to $F$, which, in turn, equals the set $\mathcal{U}_{\text {pe }}^{l c}(E, F)$ of all laterally continuous pseudo embeddings.

(3) Each laterally continuous abstract Uryson operator $T: E \rightarrow F$ is uniquely represented in the form $T=T_{D}+T_{N}$ where $T_{D}$ is a laterally continuous abstract Uryson pseudo embedding and $T_{N}$ is a laterally continuous narrow abstract Uryson operator.

Proof. Note that by [9, p. 7], $F$ is Dedekind complete. We prove $(i)$ by the following scheme

$T$ is order narrow $\stackrel{\text { Theorem } 4.1}{\Longleftrightarrow}|T|$ is order narrow $\stackrel{\text { Theorem } 5.4}{\Longleftrightarrow}|T|$ is $\lambda$-narrow

$\stackrel{\text { Theorem } 9.1}{\Longleftrightarrow}|T|$ is pseudo narrow $\stackrel{\text { by definition }}{\Longleftrightarrow} T$ is pseudo narrow.

Items (2) and (3) follow from (1) and Corollary 7.4.

\section{REFERENCES}

[1] C. D. Aliprantis, O. Burkinshaw, Positive Operators, Springer, Dordrecht. (2006).

[2] J. Flores, F. L. Hernández, P. Tradacete Domination problem for strictly singular operators and other related classes, Positivity 15, No 4 (2011), pp. 595-616.

[3] J. Flores, C. Ruiz, Domination by positive narrow operators, Positivity 7 (2003), pp. 303-321.

[4] V. M. Kadets, M. I. Kadets, Rearrangements of series in Banach spaces, Transl. Math. Mon., v.86, AMS, Providence, R.I. (1991).

[5] A. G. Kusraev, Dominated Operators, Kluwer Acad. Publ., Dordrecht-BostonLondon (2000).

[6] A. G. Kusraev, M. A. Pliev, Orthogonally additive operators on lattice-normed spaces, Vladikavkaz Math. J. No 3 (1999), pp. 33-43.

[7] A. G. Kusraev, M. A. Pliev, Weak integral representation of the dominated orthogonally additive operators, Vladikavkaz Math. J. No 4 (1999), pp. 22-39.

[8] J. Lindenstrauss, L. Tzafriri, Classical Banach spaces, Vol. 1, Sequence spaces, Springer-Verlag, Berlin-Heidelberg-New York (1977). 
[9] J. Lindenstrauss, L. Tzafriri, Classical Banach spaces. Vol.2, Function spaces, Springer-Verlag, Berlin-Heidelberg-New York (1979).

[10] O. V. Maslyuchenko, V. V. Mykhaylyuk, M. M. Popov, A lattice approach to narrow operators, Positivity 13 (2009), pp. 459-495.

[11] V. V. Mykhaylyuk, M. M. Popov, On sums of narrow operators on Kothe function spaces, J. Math. Anal. Appl. 404 (2013), pp. 554-561.

[12] J. M. MAZÓn, S. SEguRA DE LEÓN, Order bounded ortogonally additive operators, Rev. Roumane Math. Pures Appl. 35, No 4 (1990), pp. 329-353.

[13] J. M. Mazón, S. Segura de León, Uryson operators, Rev. Roumane Math. Pures Appl. 35, No 5 (1990), pp. 431-449.

[14] A. M. PlichKo and M. M. Popov, Symmetric function spaces on atomless probability spaces, Dissertationes Math. (Rozprawy Mat.) 306 (1990), pp. 1-85.

[15] M. Pliev, Uryson operators on the spaces with mixed norm, Vladikavkaz Math. J. No 3 (2007), pp 47-57.

[16] M. Pliev, Narrow operators on lattice-normed spaces, Cent. Eur. J. Math. 9, No 6 (2011), pp. 1276-1287.

[17] M. M. Popov, E. M. Semenov, D. O. VAtsek, Some problems on narrow operators on function spaces, submitted for Cent. Eur. J. Math.

[18] M. Popov, B. Randrianantoanina, Narrow Operators on Function Spaces and Vector Lattices, De Gruyter Studies in Mathematics 45, De Gruyter (2013).

South Mathematical Institute of the Russian Academy of Sciences, Str. Markusa 22, Vladikavkaz, 362027 Russia

E-mail address: maratpliev@gmail.com

Department of Applied Mathematics, Chernivtsi National University, str. KotSyUbyns'Koho 2, Chernivtsi, 58012 (Ukraine)

E-mail address: misham.popov@gmail.com 